\title{
Stringy canonical forms and binary geometries from associahedra, cyclohedra and generalized permutohedra
}

\author{
Song He, ${ }^{a, b}$ Zhenjie Li, ${ }^{a, b}$ Prashanth Raman $^{a, c, d}$ and Chi Zhang ${ }^{a, b}$ \\ ${ }^{a}$ CAS Key Laboratory of Theoretical Physics, \\ Institute of Theoretical Physics, Chinese Academy of Sciences, \\ Beijing 100190, China \\ ${ }^{b}$ School of Physical Sciences, University of Chinese Academy of Sciences, \\ No. 19A Yuquan Road, Beijing 100049, China \\ ${ }^{c}$ Institute of Mathematical Sciences, \\ Taramani, Chennai 600 113, India \\ ${ }^{d}$ Homi Bhabha National Institute, \\ Anushakti Nagar, Mumbai 400085, India \\ E-mail: songhe@itp.ac.cn, lizhenjie@itp.ac.cn, prashanthr@imsc.res.in, \\ zhangchi@itp.ac.cn
}

ABSTRACT: Stringy canonical forms are a class of integrals that provide $\alpha^{\prime}$-deformations of the canonical form of any polytopes. For generalized associahedra of finite-type cluster algebras, there exist completely rigid stringy integrals, whose configuration spaces are the so-called binary geometries, and for classical types are associated with (generalized) scattering of particles and strings. In this paper, we propose a large class of rigid stringy canonical forms for another class of polytopes, generalized permutohedra, which also include associahedra and cyclohedra as special cases (type $A_{n}$ and $B_{n}$ generalized associahedra). Remarkably, we find that the configuration spaces of such integrals are also binary geometries, which were suspected to exist for generalized associahedra only. For any generalized permutohedron that can be written as Minkowski sum of coordinate simplices, we show that its rigid stringy integral factorizes into products of lower integrals for massless poles at finite $\alpha^{\prime}$, and the configuration space is binary although the $u$ equations take a more general form than those "perfect" ones for cluster cases. Moreover, we provide an infinite class of examples obtained by degenerations of type $A_{n}$ and $B_{n}$ integrals, which have perfect $u$ equations as well. Our results provide yet another family of generalizations of the usual string integral and moduli space, whose physical interpretations remain to be explored.

KEYwords: Scattering Amplitudes, Differential and Algebraic Geometry

ARXIV EPRINT: 2005.07395 


\section{Contents}

1 Introduction $\quad 1$

1.1 Summary of main results 4

2 Configuration spaces with perfect $u$ equations from degenerating $\mathscr{A}_{n}$ and $\begin{array}{ll}\mathscr{B}_{n} & \mathbf{7}\end{array}$

$2.1 \mathscr{A}_{n}$ and $\mathscr{B}_{n}$ as generalized permutohedra $\quad 7$

2.2 Degenerations of $\mathscr{A}_{n}$ with perfect $u$ equations $\quad 9$

$\begin{array}{lll}2.3 & \text { Degenerations of } \mathscr{B}_{n} \text { and products } & 14\end{array}$

$\begin{array}{lll}\text { 2.3.1 Degenerations of } \mathscr{B}_{n} & 14\end{array}$

$\begin{array}{lll}2.3 .2 & \text { Products of } \mathscr{A} \text { and } \mathscr{B} & 16\end{array}$

3 Stringy canonical forms and binary geometries for generalized permutohedra

$\begin{array}{lll}3.1 & \text { Generalized permutohedra } & 17\end{array}$

$\begin{array}{lll}3.2 & \text { ABHY-like realizations and } u \text { variables for generalized permutohedra } & 19\end{array}$

$\begin{array}{ll}3.3 & \text { Permutohedra as binary geometries } \\ & 22\end{array}$

3.3.1 The $u$ equations of $\mathscr{P}_{n} \quad 23$

3.4 Generalized permutohedra as binary geometries 25

$\begin{array}{llr}4 & \text { Discussions } & 28\end{array}$

$\begin{array}{ll}\text { A Degenerations of } \mathscr{P}_{n} \text { which lead to products } & 31\end{array}$

B Some $u$ equations of $\mathscr{P}_{n}$

\section{Introduction}

In [1] the notion of stringy canonical forms was introduced which provides vast generalizations of tree-level string amplitudes as integrals over the moduli space $\mathcal{M}_{0, n} \cdot{ }^{1}$ Various nice properties of string amplitudes and their field-theory limits, such as factorizations for $\alpha^{\prime} \rightarrow 0$ limit and finite $\alpha^{\prime}$, channel duality and exponential softness at UV, scattering equations, etc. all naturally extend to stringy canonical forms. To briefly summarize the idea, let's begin with the disk integral for the open-string amplitudes with ordering $(1,2, \ldots, n)$ :

$$
\mathcal{I}_{1,2, \ldots, n}^{\text {open-string }}(\{s\}):=\int_{\mathcal{M}_{0, n}^{+}} \frac{\mathrm{d}^{n} z}{\operatorname{SL}(2, \mathbb{R})} \prod_{i=1}^{n} \frac{1}{z_{i}-z_{i+1}} \prod_{i<j}\left(z_{j}-z_{i}\right)^{\alpha^{\prime} s_{i j}},
$$

\footnotetext{
${ }^{1}$ The study of these types of integrals perhaps dates back to Euler, and more recently they are studied in various contexts, see e.g. [2-10].
} 
where $\mathcal{M}_{0, n}^{+}$is the positive part of moduli space for the ordering, and $\mathrm{PT}_{n}:=$ $\frac{\mathrm{d}^{n} z}{\mathrm{SL}(2, \mathbb{R})} \prod_{i=1}^{n} \frac{1}{z_{i}-z_{i+1}}$ its canonical form [11]. With a positive parametrization $\left\{x_{1}, \ldots, x_{n-3}\right\}$ of $\mathcal{M}_{0, n}^{+}$, this canonical form becomes $\prod_{i=1}^{n-3} \mathrm{~d} \log x_{i}$, and the Koba-Nielsen factor $\prod_{i<j}\left(z_{j}-\right.$ $\left.z_{i}\right)^{\alpha^{\prime} s_{i j}}$ becomes a product of powers of polynomials with non-negative coefficients [1]: $\prod_{i} x_{i}^{\alpha^{\prime} X_{i}} \prod_{I} p_{I}(x)^{-\alpha^{\prime} c_{I}}$. Note that we have factored out $n-3$ monomials, $x_{i}$, and left with $n(n-3) / 2-(n-3)$ positive polynomials $p_{I}(x)$, with exponents $X_{i}$ 's and $c_{I}$ 's given by linear combinations of the Mandelstam variables $s_{i j}$.

Stringy canonical forms. Motivated by this rewriting of string integral (1.1), we define a general stringy integral as a $d$-dimensional integral [1]

$$
\mathcal{I}(\mathbf{X},\{c\})=\int_{\mathbb{R}_{+}^{d}} \prod_{i=1}^{d} \frac{\mathrm{d} x_{i}}{x_{i}} x_{i}^{\alpha^{\prime} X_{i}} \prod_{I=1}^{m} p_{I}(x)^{-\alpha^{\prime} c_{I}},
$$

where we allow $m$ arbitrary Laurent polynomials with non-negative coefficients, $p_{I}(x)$, and without loss of generality we assume exponents $X_{i}>0$ and $c_{I}>0 .^{2}$

Any integral of the form (1.2) inherits various remarkable properties of string amplitudes (1.1), which all depends on the existence of a polytope $\mathcal{P}:=\sum_{I} c_{I} N\left[p_{I}\right]$; here $N\left[p_{I}\right]$ is the Newton polytope of the polynomial $p_{I}$, and we take the (weighted) Minkowski sum [1]. First of all, one can show that the integral (1.2) is convergent if and only if $\mathbf{X}=\left(X_{1}, \ldots, X_{d}\right)$ is in the interior of the polytope $\mathcal{P}$ (we assume that $\mathcal{P}$ is $d$ dimensional). The leading order of the integral in its $\alpha^{\prime}$ expansion, dressed with $\mathrm{d}^{d} \mathbf{X}$, is given by the canonical form of the polytope, $\Omega(\mathcal{P})$, thus it can be viewed as an $\alpha^{\prime}$-deformation of $\Omega(\mathcal{P})$ (hence the name "stringy canonical form"). Generically the poles of $\Omega(\mathcal{P})$ are given by equations of facets for $\mathcal{P}$, which are also poles of the integral at finite $\alpha^{\prime}$, and the residue on any such pole is given by a stringy canonical form defined for that facet (see [1] for details).

For the string integral (1.1), $\mathcal{P}$ is nothing but the ABHY associahedron [11], whose canonical form gives bi-adjoint $\phi^{3}$ amplitude as the $\alpha^{\prime} \rightarrow 0$ limit of (1.1) [12]. The poles are the planar variables $X_{i, j}$, on which (1.1) factorizes into lower-point integrals even for finite $\alpha^{\prime}$. Moreover, the saddle point equations as $\alpha^{\prime} \rightarrow \infty$ can be written as $X_{i}=$ $\sum_{I} c_{I} \partial \log p_{I} / \partial \log x_{i}$ for $i=1, \ldots, d$; they provide a diffeomorphism from the integration domain to $\mathcal{P}$, thus $\Omega(\mathcal{P})$ can be computed as a pushforward of $\prod_{i} \mathrm{~d} \log x_{i}$ via summing over the saddle points [13]. In particular, for the string integral (1.1), the scattering equations provide a map from $\mathcal{M}_{0, n}^{+}$to $\mathrm{ABHY}$ associahedron, and the pushforward is equivalent to the CHY formula for $\phi^{3}$ amplitude $[12,14]$.

Configuration spaces. One way to describe the combinatorics of the polytope $\mathcal{P}$ of the stringy integral (1.2) is through the configuration space, e.g. as a blow-up of the integration domain, $\mathbf{x}:=\left(x_{1}, \ldots, x_{d}\right)$-space $\mathbb{R}_{+}^{d}$. However, it is usually difficult to use $\mathbf{x}$ to describe the polytope $\mathcal{P}$, even for the string integrals (1.1). For example, a facet usually corresponds to a complicated limit process with $x \rightarrow 0$ or $\infty$. As proposed in [15], it's more

\footnotetext{
${ }^{2}$ These are "positive" stringy canonical forms, which are integrals over $\mathbb{R}_{+}^{d}$. We can also consider "complex" cases with the integrand mod-squared and integrated over $\mathbb{C}^{d}$, which are generalizations of closedstring amplitudes [1].
} 
elegant to realize $\mathcal{P}$ by introducing a set of constrained $u$ variables $\left\{u_{\alpha}\right\}$ which satisfy a set of polynomial equations called $u$ equations. The configuration space is the variety of $u$ variables defined by $u$ equations, where the polytope $\mathcal{P}$ can be realized combinatorially by $\left\{u_{\alpha} \geq 0\right\}$ such that each facet $F_{\alpha}$ of $\mathcal{P}$ is simply given by an equation $u_{\alpha}=0$ and its interior is described by $\left\{u_{\alpha}>0\right\}$. To define the corresponding $u$ variables of the stringy canonical form (1.2) from $\mathbf{x}$-space $\mathbb{R}_{+}^{d}$, we first construct the so-called big polyhedron in the $\mathbf{S}:=\left(X_{1}, \ldots, X_{d},-c_{1}, \ldots,-c_{m}\right)$-space $\mathbb{R}^{d+m}$. The polytope $\mathcal{P}$ in the space of $\mathbf{X}:=\left(X_{1}, \ldots, X_{d}\right)$ is bounded by inequalities $W_{a}^{J} S_{J} \geq 0$, where $W_{a}^{J}$ is determined by the facet $F_{a}$ of $\mathcal{P}$. These inequalities also cut out a polyhedron in the $(d+m)$-dimensional space of $\mathbf{S}$, which is defined to be the big polyhedron of $\mathcal{P}$, and the integral $\mathcal{I}(\mathbf{S})$ converges for each point $\mathbf{S}$ inside this polyhedron. Dually, we can describe the big polyhedron by its vertices. Suppose $\left\{V_{J}^{\alpha}\right\}_{1 \leq \alpha \leq v}$ are its non-zero vertices ( $v$ denotes the number of vertices), a point $\mathbf{S}$ inside the polyhedron can be written as $S_{J}=F_{\alpha} V_{J}^{\alpha}$ with non-negative coefficients $\left\{F_{\alpha}\right\}_{1 \leq \alpha \leq v}$. Therefore, the integral $\mathcal{I}(\mathbf{S})$ can be seen as a function of "dual coordinates" $\left\{F_{\alpha}\right\}$ which encourages us to rewrite the integral to make it manifest by (here we collectively denote $\left.p_{J}=\left(x_{1}, \cdots, x_{d}, p_{1}, \cdots, p_{m}\right)\right)^{3}$

$$
\mathcal{I}(\mathbf{F})=\int_{\mathbb{R}_{+}^{d}} \prod_{i=1}^{d} \frac{d x_{i}}{x_{i}} \prod_{\alpha} u_{\alpha}^{\alpha^{\prime} F_{\alpha}}=\int_{\mathbb{R}_{+}^{d}} \prod_{i=1}^{d} \frac{d x_{i}}{x_{i}} \prod_{J}^{d+m} p_{J}^{\alpha^{\prime} S_{J}},
$$

where we introduce a new set of variables $u_{\alpha}=\prod_{J} p_{J}^{V_{J}^{\alpha}}$ for $\alpha=1, \ldots, v$. Note that there's no unique way to write $S_{J}=F_{\alpha} V_{J}^{\alpha}$ if the big polyhedron is not a simplex, i.e. $v>d+m$, and in this case, $u$ variables $\left\{u_{\alpha}\right\}$ are not multiplicatively independent. Therefore, it is an important special case when the big polyhedron is a simplex, i.e. $N=v=d+m$, where $N$ is the number of facets of the big polyhedron or the original polytope $\mathcal{P}$, and inequalities $F_{\alpha}=S_{J}\left(V^{-1}\right)_{\alpha}^{J} \geq 0$ are exactly the inequalities $W_{a}^{J} S_{J} \geq 0$ up to rescaling $F_{\alpha} \mapsto t F_{\alpha}$ for positive factors $t$, because there's no nontrivial linear isometry from the simplex to itself besides permutations of vertices. Therefore, we identity the label of facets $\alpha$ with the label of vertices $J$ and get $V=W^{-1}$. Furthermore, every facet of the original polytope $\mathcal{P}$ can be associated with a single $u_{\alpha}$ going to zero.

Cluster stringy integrals and binary geometries. A class of very special integrals belong to this case [15]: stringy canonical forms for generalized associahedra of finite-type cluster algebras, namely $A_{n}, B_{n}, C_{n}, D_{n}$, and exceptional cases $\left(E_{6}, E_{7}, E_{8}, F_{4}, G_{2}\right)$. These "cluster string integrals" [15], and the associated "cluster configuration spaces" $[15,16]$, turn out to be much more special and rigid extensions of string amplitudes (1.1) and the moduli space $\mathcal{M}_{0, n}$, which correspond to the case of type $A_{n-3}$. The leading order of such an integral is given by the canonical function of the corresponding generalized associahedra, whose combinatorics is attached to the Dynkin diagram (each facet is given by removing a node), e.g. $D_{n} \rightarrow D_{m} \times A_{n-1-m}$ or $A_{n-1}$ [15]. Even at finite $\alpha^{\prime}$, it has the

\footnotetext{
${ }^{3}$ Note that one can indeed treat the monomials $x_{i}^{\alpha^{\prime} X_{i}}$ on the equal footing as polynomials, since Minkowski sum w.r.t. them amounts to merely translating the polytope $c_{I} N\left[p_{I}\right]$ by $-X_{i}$ in the $i$-th direction [1].
} 
remarkable factorization property tied to the Dynkin diagram. Moreover, for type $B / C$ (known as "cyclohedron") and $D$, the leading orders are associated with tadpole emission and one-loop bi-adjoint $\phi^{3}$ amplitudes, respectively [17, 18].

These nice properties become manifest in the configuration space: not only the big polyhedron is a simplex, the $u$ equations are of very special form:

$$
1-u_{\alpha}=\prod_{\beta} u_{\beta}^{\beta \| \alpha}
$$

where the product is over all $N$ variables ( $N$ facets), and the integer $\beta \| \alpha \geq 0$ is the so-called compatibility degree defined in the corresponding cluster algebra [19]. Varieties defined by $u$ equations associated with finite type clusters are called cluster configuration spaces. We see that when $u_{\alpha}$ (for a facet $\alpha$ ) goes to 0 , it forces all the $u_{\beta}$ with $\beta \| \alpha>0$ to go to 1 (for facets $\beta$ are exactly those incompatible with $\alpha$ ); the remaining $u$ 's that are compatible with $u_{\alpha}$ satisfy $u$ equations for the facet, which factorizes as removing a node of the Dynkin diagram. Therefore, we say that the configuration space is a binary geometry. Note that this is true also for the complex case, and if we further require all $u$ variables to be positive, we have $0<u<1$ which gives the positive configuration space [20]. The binary property is what guarantees the stringy integral to factorize even at finite $\alpha^{\prime}$ : on the pole $X_{\alpha} \rightarrow 0, u_{\alpha} \rightarrow 0$ forces all incompatible $u_{\beta} \rightarrow 1$ which are decoupled from $\prod u^{\alpha^{\prime} X}$, and the integral becomes the factorized one for the facet $\alpha$.

For example, the string integral (1.1) corresponds to type $A_{n-3}$, and the $N=n(n-3) / 2$ $u$ variables of this configuration space are [20]

$$
u_{i j}=\frac{\left(z_{i-1}-z_{j}\right)\left(z_{i}-z_{j-1}\right)}{\left(z_{i}-z_{j}\right)\left(z_{i-1}-z_{j-1}\right)}
$$

and they satisfy the $u$ equations (see e.g. [5])

$$
1-u_{i j}=\prod_{(k, l) \nsim(i, j)} u_{k l}
$$

where $(i, j) \nsim(k, l)$ means that the diagonals $(i, j)$ and $(k, l)$ of $n$-gon are crossed, thus the two facets are incompatible (note here compatibility degree is just 0 or 1 ). For $u_{i, j} \rightarrow 0$, all incompatible $u_{k, l} \rightarrow 1$, and the remaining $u$ 's fall into those of the two polygons divided by $(i, j)$, with their own $u$ equations. The Koba-Nielsen factor becomes $\prod_{i, j} u_{i, j}^{\alpha^{\prime} X_{i, j}}$ where $X_{i, j}$ are planar variables for the facets of $\mathrm{ABHY}$ associahedron, which makes the factorization of string amplitude at finite $\alpha^{\prime}$ manifest. All these generalize to integrals and configuration spaces for finite type cluster algebras.

\subsection{Summary of main results}

It is a natural question to ask if binary geometries, whose stringy integrals factorize at any finite $\alpha^{\prime}$, are extremely special and can only be products of generalized associahedra of finite type. In this paper we answer the question in the negative by providing infinitely many new examples of binary geometries. Before proceeding, we remark that $u$ equations 
of the form (1.4) (which we refer to as "perfect" $u$ equations) imply binary geometries, but the converse is not true. Broadly speaking, for a $n$-dimensional polytope $\mathcal{P}$ where each facet is associated with a $u$ variable, if for any $u_{\alpha} \rightarrow 0$, we have for all incompatible facets $\beta, u_{\beta} \rightarrow 1$, then such an $n$-dimensional variety (defined by the $u$ equations) is a binary geometry. From this definition, we see that the simplest example of binary geometry is a simplex, where all facets are compatible with each other. One can realize the configurations space by the following $u$ equations for a $n$-dimensional simplex with facets labelled by $\alpha=0,1, \ldots, n$ :

$$
1-u_{\alpha}=\sum_{\beta \neq \alpha} u_{\beta}
$$

As $u_{\alpha} \rightarrow 0$, there is no $u \rightarrow 1$, and all $n$ remaining $u$ variables satisfy the same equations for that facet, which is an $(n-1)$-dimensional simplex. Although this example is trivial, it does show that we do not necessarily need perfect $u$ equations to have binary geometries. Generically, a $n$-dimensional polytope $\mathcal{P}$ with $N$ facets have an $n$-dimensional configuration space defined by $u$ equations

$$
1-u_{\alpha}=p_{\alpha}(\{u\}) \prod_{\beta} u_{\beta}^{\beta \| \alpha}
$$

where $\beta \| \alpha \geq 0$ are integers which vanishes for $\beta$ compatible with $\alpha$, and $p_{\alpha}(\{u\})$ is an arbitrary polynomial which must equal to unity on the facet $\alpha,\left.p_{\alpha}(\{u\})\right|_{u_{\alpha}=0}=1$. When $u_{\alpha} \rightarrow 0$, since it appears on the r.h.s. of the equation with $1-u_{\beta}$ for any incompatible $\beta$, we have $u_{\beta} \rightarrow 1$ for any polynomial $p_{\beta}(\{u\})$. However, in the equation with $1-u_{\alpha}$, since all the incompatible $u_{\beta} \rightarrow 1$ we find $\left.p_{\alpha}(\{u\})\right|_{u_{\alpha}=0}=1$ (where we evaluate the polynomial at $u_{\alpha}=0$ and all incompatible $u_{\beta}=1$ ). For cluster configuration spaces, we have perfect $u$ equations with $p_{\alpha}=1$ for all $\alpha$.

As the main result of the paper, we present infinitely many new examples of binary geometries which have $u$ equations of the form (1.6), and (also infinitely) many of them with perfect $u$ equations. The key idea to consider a class of stringy integrals for generalized permutohedra that can be realized as the Minkowski sum of coordinate simplices is as follows (more details will be given later). Given the label set $[0, n]=\{0,1, \ldots, n\}$, we define a building set $\mathbf{B}$ as a collection of subsets $I \subset[0, n]$ that satisfy (1). if $I, J \in \mathbf{B}$ and $I \cap J \neq \varnothing$, then $I \cup J \in \mathbf{B}$, and (2). it contains all singletons $\{i\}$. For each subset $I$ we have a simplex $\Delta_{I}$ as the convex hull of unit vectors $\mathbf{e}_{i}$ with $i \in I$, and the generalized permutohedron $\mathscr{P}(\mathbf{B})$ is given by (weighted) Minkowski sum of $\Delta_{I}$ (defined on a $n$-dimensional hyperplane of the $(n+1)$-dimensional space) [21]. It is very natural to define stringy canonical form for such a building set $\mathbf{B}$ : for each subset $I$, we have the simplest linear polynomial $x_{I}:=\sum_{i \in I} x_{i}$ (for singletons, $x_{\{i\}}=x_{i}$ are the coordinates), and

$$
\mathcal{I}_{\mathbf{B}}(\{S\}):=\left(\alpha^{\prime}\right)^{n} \int_{\mathbb{R}_{+}^{n}} \frac{1}{\operatorname{vol} \operatorname{GL}(1)} \prod_{i=0}^{n} \frac{d x_{i}}{x_{i}} \prod_{I \in \mathbf{B}} x_{I}^{\alpha^{\prime} S_{I}},
$$

where the integration domain is defined projectively, i.e. up to a GL(1) redundancy thus the exponents $S_{I}$ satisfy $\sum_{I} S_{I}=0$ (one can fix the gauge by choosing e.g. $x_{0}=1$, then 
$S_{0}$ drops out and the remaining $S_{I}$ are independent). By construction, $\mathcal{I}_{\mathbf{B}}$ is a stringy canonical form for the generalized permutohedron $\sum_{I} S_{I} \mathbf{N}\left[x_{I}\right]=\sum_{I} S_{I} \Delta_{I}=\mathscr{P}(\mathbf{B})$, and the leading order is given by the volume of its dual. Note that one can split the product as $\prod_{I} x_{I}^{\alpha^{\prime} S_{I}}=\prod_{i=1}^{n} x_{i}^{\alpha^{\prime} X_{i}} \prod_{I}^{m} x_{I}^{-\alpha^{\prime} C_{I}}$ with $X_{i}:=S_{\{i\}}$ for singletons, $-C_{I}=S_{I}$ for nonsingleton $I$ 's (we denote their total number as $m$ ), and it suffices to take the Minkowski sum w.r.t. the non-singleton I's, since the singleton ones are merely translating the polytope. Let's first present the simplest example with building set $\mathbf{B}$ given by all the singletons $\{i\}$ and $[0, n]$. In this case, $\mathscr{P}(\mathbb{B})=c \mathbf{N}\left[\sum_{i=0}^{n} x_{i}\right]$ is the $n$-dimensional simplex (we denote $c:=$ $\left.C_{[0, n]}\right)$, given by $X_{i} \geq 0$ with one (ABHY) constraint $\sum_{i=1}^{n} X_{i}=c$. It is straightforward to see that the $u$ variables are $u_{i}=\frac{x_{i}}{x_{[0, n]}}=\frac{x_{i}}{\sum_{i} x_{i}}$ and they satisfy $\sum_{i=0}^{d} u_{i}=1$ as expected. In this case, the integral is trivial to perform, which yields a generalization of beta function to higher dimensions:

$$
\mathcal{I}_{n}^{\text {simplex }}=\frac{\prod_{i=1}^{n} \Gamma\left(\alpha^{\prime} X_{i}\right) \Gamma\left(\alpha^{\prime}\left(c-\sum_{i} X_{i}\right)\right)}{\Gamma\left(\alpha^{\prime} c\right)}:=B\left(\alpha^{\prime} X_{1}, \ldots, \alpha^{\prime} X_{n}, \alpha^{\prime}\left(c-\sum_{i} X_{i}\right)\right) .
$$

The most important three examples of $\mathscr{P}(\mathbf{B})$ and $\mathcal{I}_{\mathbf{B}}$, which will be studied in detail throughout the paper, are summarized here:

- Consider $\mathbf{B}$ as the collection of all $n(n+3) / 2+1$ consecutive intervals of $[0, n]$, $\mathscr{P}(\mathbf{B}) \equiv \mathscr{A}_{n}$ is the $n$-dimensional associahedron, (1.7) is nothing but $(n+3)$-pt string integral (1.1); e.g. for $n=2, \mathbf{B}=\{\{i\},\{0,1\},\{1,2\},\{0,1,2\}\}$, and we have a pentagon $\mathscr{A}_{2}$.

- Consider $\mathbf{B}$ as the collection of all $n(n+1)+1$ cyclic intervals of $[0, n]$ which can wrap around $n, \mathscr{P}(\mathbf{B}) \equiv \mathscr{B}_{n}$ is the $n$-dimensional cyclohedron, and in section 2 we will show that non-trivially (1.7) is the cluster stringy integral for type $B_{n}$ (not that for type $C_{n}$ ); e.g. $n=2, \mathbf{B}=\{\{i\},\{0,1\},\{1,2\},\{2,0\},\{0,1,2\}\}$, and we have a hexagon $\mathscr{B}_{2}$.

- Consider $\mathbf{B}$ as the collection of all $2^{n+1}-1$ nonempty subsets of $[0, n], \mathscr{P}(\mathbf{B}) \equiv \mathscr{P}_{n}$ is the $n$-dimensional permutohedron (for $n=2$, it coincides with $\mathscr{B}_{2}$ ). All generalized permutohedra (and their stringy integrals) can be obtained from (that of) $\mathscr{P}_{n}$.

Although associahedra and cyclohedra are special cases of generalized permutohedra, it is interesting that (1.7) in these cases turn out to be exactly cluster stringy integrals of type $A$ and $B$ ! It is not completely surprising since these are the only two cases of cluster configuration spaces that can be identified with hyperplane arrangement [16], which is consistent with the fact that here we only have linear polynomials $x_{I}$. In section 2 , we will go further and discover more binary geometries with perfect $u$ equations, which can be obtained by "degenerating" type $A$ and $B$ integrals by requiring certain $S_{I}=0$ (for example, type $A$ integral is in fact a degeneration of the type $B$ integral, which was not obvious before). Some of these cases are products of lower-dimensional $A$ 's and $B$ 's, but we will see entirely new binary geometries with perfect $u$ equations.

Moreover, a remarkable statement we will make in section 3 is that, the configuration space for any $\mathcal{I}_{\mathbf{B}}$ is always binary, which means that any $u_{\alpha} \rightarrow 0$ forces all the incompatible 
ones $u_{\beta} \rightarrow 1$. As we will see explicitly with $\mathscr{P}_{n}$, its $u$ equations indeed take the form of (1.6), which is no longer perfect for $n>2$, but the space is binary and the integral factorizes as a product of lower-dimensional permutohedron integrals at finite $\alpha^{\prime}$ ! Note that an important feature of any generalized permutohedron is that its facets are labelled by all subsets $I$ in B except for the complete set $[0, n]$, and on each facet it factorizes as a product of two generalized permutohedra. The total number of facets $N$ equals $|\mathbf{B}|-1=n+m$, thus the big polyhedron is indeed a simplex as expected. In section 3, we will present the formula for $u$ variables for any generalized permutohedron (which is equivalent to the formula for its ABHY-like realization), from which the binary and factorization properties follow.

\section{Configuration spaces with perfect $u$ equations from degenerating $\mathscr{A}_{n}$ and $\mathscr{B}_{n}$}

In this section we shall consider some degenerations of $n$-dimensional associahedron $\mathscr{A}_{n}$ and $n$-dimensional cyclohedron $\mathscr{B}_{n}$ and show that there are an infinite class of examples of binary geometries with perfect $u$ equations. To do this we shall use the fact that both $\mathscr{A}_{n}$ and $\mathscr{B}_{n}$ can be realised as a Minkowski sum of coordinate simplices.

\section{1 $\mathscr{A}_{n}$ and $\mathscr{B}_{n}$ as generalized permutohedra}

Associahedron $\mathscr{A}_{\boldsymbol{n}}$. The building set of $\mathscr{A}_{n}$ is $\mathbf{B}=\{[i, j]: 0 \leq i \leq j \leq n\}$. The stringy canonical form of this building set $\mathcal{I}_{\mathbf{B}}(\{S\})$ is related with the original string integral eq. (1.1) by

$$
z_{j}-z_{i}=x_{[i, j-1]},
$$

so Mandelstam variables are related to $S$ by $s_{i j}=S_{[i, j-1]}$. The facets of $\mathscr{A}_{n}$ are given by

$$
F_{[i, j]}=\sum_{[k, l] \subset[i, j]} S_{[k, l]},
$$

and their solution is (a rewriting of ABHY conditions in our notation):

$$
S_{[i, j]}=F_{[i, j]}+F_{[i+1, j-1]}-F_{[i, j-1]}-F_{[i+1, j]},
$$

where $F_{[k, l]}:=0$ when $k>l$ or $[k, l]=[0, n]$ and the usual planar $X$ variables $X_{i j}$ is $F_{[i, j-2]}$. Using this solution to rewrite the integrand of the stringy canonical form $\mathcal{I}_{\mathbf{B}}(\{S\})$

$$
\prod_{0 \leq i \leq j \leq n} x_{[i, j]}^{S_{[i, j]}}=\prod_{0 \leq i \leq j \leq n} x_{[i, j]}^{F_{[i, j]}} x_{[i, j]}^{F_{[i+1, j-1]}} / x_{[i, j]}^{F_{[i, j-1]}} x_{[i, j]}^{F_{[i+1, j]}}
$$

and we get that the $u$ variables easily:

$$
u_{i, j+2}:=u_{[i, j]}=\frac{x_{[i, j]} x_{[i-1, j+1]}}{x_{[i, j+1]} x_{[i-1, j]}} \quad \text { for } 0 \leq i \leq j \leq n,
$$

with $x_{[i, n+1]}:=1$ and $x_{[-1, j]}:=1$, and it is straightforward to see that they satisfy $u$ equations (1.5). 
The ABHY construction of $\mathscr{A}_{n}$ is given by

$$
S_{[i, j]}=-C_{[i, j]} \text { for all } i<j, \quad F_{[i, j]} \geq 0 \text { for all } i \leq j,
$$

where $C$ 's are positive numbers. As first introduced in [18, 22], it is convenient and illuminating to represent these conditions in a mesh diagram of $(1+1)$-D lattice. For example, we can have the following mesh for ABHY realization of $\mathscr{A}_{3}$ :

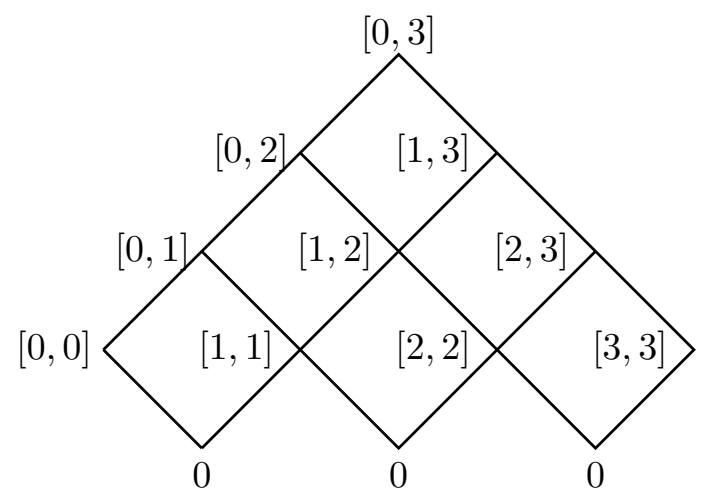

where each diamond is labelled by $[i, j]$ of the top corner, ${ }^{4}$ and it gives

$$
C_{[i, j]}=F_{[i, j-l]}+F_{[i+1, j]}-F_{[i, j]}-F_{[i+1, j-1]} \cdot
$$

For each causal diamond, we have the Gauss law:

$$
\sum_{\substack{i \leq a<j \\ k<b \leq l}} c_{[a, b]}=F_{[i, k]}+F_{[j, l]}-F_{[i, l]}-F_{[j, k]}
$$

where $F_{[a, b]}:=0$ when $a>b$ or $[a, b]=[0, n]$

Cyclohedron $\mathscr{B}_{n}$. The building set of $\mathscr{B}_{n}$ is $\mathbf{B}=\{[i, i+k]: 0 \leq i, k \leq n\}$ where the labels are understood to be $\bmod n+1$. The facets are given by

$$
F_{[i, j]}=\sum_{[k, l] \subset[i, j]} S_{[k, l]},
$$

and their solution is

$$
S_{[i, i]}=F_{[i, i]}, \quad S_{[i, j]}=F_{[i, j]}+F_{[i+1, j-1]}-F_{[i, j-1]}-F_{[i+1, j]} \quad \text { for } i \neq j \text { and }[i, j] \neq[0, n],
$$

and

$$
S_{[0, n]}=F_{[0, n]}-\sum_{i=0}^{n} F_{[i, i-2]}+\sum_{i=0}^{n} F_{[i, i-3]},
$$

where $F_{[i+1, i]}=F_{[0, n]}=0$. Therefore, the $u$ variables are

$$
u_{i}:=u_{[i, i-2]}=\frac{x_{[i, i-2]}}{x_{[0, n]}}, \quad u_{i, j+2}:=u_{[i, j]}=\frac{x_{[i, j]} x_{[i-1, j+1]}}{x_{[i, j+1]} x_{[i-1, j]}} \quad \text { for } i-j \neq 2 .
$$

\footnotetext{
${ }^{4}$ It looks different with the usual mesh of $\mathscr{A}_{n}$ [18] where label of $c$ follows the left corner in each diamond. This is because the mismatch of the correspondence of labels $X_{i j}=F_{[i, j-2]}$ and $s_{i j}=S_{[i, j-1]}$.
} 
It is straightforward to work out the $u$ equations, which can be put in a compact form [15]

$$
1-u_{i}=U_{[i+1, i-1]}, \quad 1-u_{i j}=U_{[i+1, j],[j+1, i-1]} U_{[j+1, i-1],[i, j-1]} U_{[j+1, i-1]}^{2},
$$

where we introduce the notation

$$
U_{A, B}=\prod_{a \in A, b \in B} u_{a b}, \quad U_{A}=\prod_{a \in A} u_{a} \prod_{a<b \in A} u_{a b} .
$$

Since the building set of $\mathscr{A}_{n}$ is contained in the building set of $\mathscr{B}_{n}, \mathscr{A}_{n}$ is a degeneration of $\mathscr{B}_{n}$, which was not obvious in the previous construction of $\mathscr{B}_{n}$.

The ABHY construction of $\mathscr{B}_{n}$ is similar: $F_{[i, j]} \geq 0$ for all $[i, j] \in \mathbf{B}$ and for $i \neq j$

$F_{[i, j-1]}+F_{[i+1, j]}-F_{[i, j]}-F_{[i+1, j-1]}=C_{[i, j]}$ for $[i, j] \neq[0, n], \quad C_{[0, n]}=\sum_{i=0}^{n} F_{[i, i-2]}-\sum_{i=0}^{n} F_{[i, i-3]}$,

where $F_{[i+1, i]}=0$ and the $C$ 's are all positive constants.

Like $A_{n}, B_{n}$ type cluster stringy canonical form also comes from a hyperplane arrangement, the Shi arrangement [23]. The Shi arrangement contains $n+1$ punctures $\left\{z_{i}\right\}_{i=1, \ldots, n+1}$ on the real line with the freedom of global transformation $z_{i} \rightarrow z_{i}+a$ which can be used to fix $z_{n+1}=0$. The Shi arrangement is given by the following hyperplanes

$$
z_{i}-z_{j}=0 \quad \text { and } \quad z_{i}-z_{j}=1 \quad \text { for } 1 \leq i<j \leq n+1,
$$

and its stringy integral is

$$
\mathcal{I}_{n}=\int_{1>z_{1}>z_{2}>\cdots>z_{n}>0} \frac{\mathrm{d} z_{1} \cdots \mathrm{d} z_{n}}{\left(1-z_{1}\right)\left(z_{1}-z_{2}\right) \cdots\left(z_{n}-0\right)} \prod_{1 \leq i<j \leq n+1}\left(z_{i}-z_{j}\right)^{s_{i j}}\left(1-z_{i}+z_{j}\right)^{t_{i j}},
$$

where the positive region is given by $0<z_{i}-z_{j}<1$ for $1 \leq i<j \leq n+1$. We can also write the $u$ variables in terms of the $z$ 's:

$$
\begin{aligned}
& u_{1}=\tilde{z}_{1}-z_{2}, \quad \ldots, \quad u_{n}=\tilde{z}_{n}-z_{n+1}, \quad u_{n+1}=z_{n+1}-z_{1}, \\
& u_{j i}=\frac{\left(z_{j+1}-z_{i}\right)\left(z_{i+1}-z_{j}\right)}{\left(z_{j+1}-z_{i+1}\right)\left(z_{i}-z_{j}\right)} \quad \text { for } i<j, \\
& u_{i j}=\frac{\left(\tilde{z}_{j+1}-z_{i}\right)\left(\tilde{z}_{i+1}-z_{j}\right)}{\left(\tilde{z}_{j+1}-z_{i+1}\right)\left(\tilde{z}_{i}-z_{j}\right)} \quad \text { for } i<j,
\end{aligned}
$$

where $\tilde{z}_{i}=z_{i+n+1}=z_{i}+1$ and indices live in $\mathbb{Z}_{2 n+2}$.

\subsection{Degenerations of $\mathscr{A}_{n}$ with perfect $u$ equations}

The degenerations of $\mathscr{A}_{n}$ are given by setting some $C$ 's in ABHY construction to zero or deleting the corresponding elements in the building set. Note that the building set after deletion doesn't need to be a new building set, i.e. a degeneration of $\mathscr{A}_{n}$ may not be a generalized permutohedron. However, we can still ask the following question: are these degenerations binary geometries? Of course, we should require at least that the number of 
facets of the degeneration of $\mathscr{A}_{n}$ should be equal to the size of the corresponding deleted building set minus one, i.e. its big polyhedron is a simplex. Before answering this question, let's first try to find all degenerations of $\mathscr{A}_{n}$ with this property.

From the following equations for facets $\left\{F_{[i, j]}\right\}$ of $\mathscr{A}_{n}$

$$
F_{[i, k]}+F_{[j, l]}-F_{[i, l]}-F_{[j, k]}=\sum_{\substack{i \leq a<j \\ k<b \leq l}} C_{[a, b]}
$$

where $F_{[a, b]}:=0$ when $a>b$ or $[a, b]=[0, n]$, a standard way to get a degeneration whose big polyhedron is a simplex is setting all $C$ 's on the r.h.s. of

$$
\begin{aligned}
F_{[i, j-1]}+F_{[j, l]}-F_{[i, l]} & =\sum_{i \leq a<j \leq b \leq l} C_{[a, b]} & & \text { for } i<j=k+1 \leq l \\
F_{[0, k]}+F_{[j, n]}-F_{[j, k]} & =\sum_{\substack{0 \leq a<j \\
k<b \leq n}} C_{[a, b]} & & \text { for } 0<j \leq n \text { and } 0 \leq k<n
\end{aligned}
$$

to be zero, and all wanted degenerations can be got by setting $C$ 's to zero in several equations above.

From the viewpoint of the mesh picture, an equation above corresponds to a big diamond whose top or bottom corner and all $C$ 's are zero. For example,
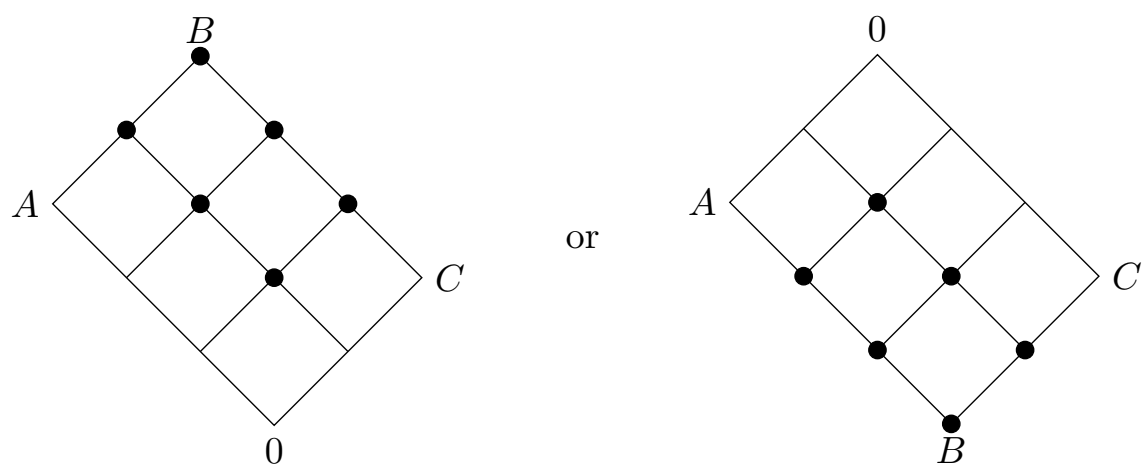

we can see that

$$
A+C-B=0
$$

Since $A, B, C \geq 0$, the above equation forces $A$ and $C$ to vanish when $B=0$. Therefore, $B$ is no longer a facet of the degeneration. Similarly, black points are all vanishing facets in the above example, and generally the number of vanishing facets equals the number of deleted $C$ 's for degenerations of this type.

One can use these diamonds to build a degeneration with more vanishing facets whose big polyhedron is a simplex. For example, in $\mathscr{A}_{3}$ 


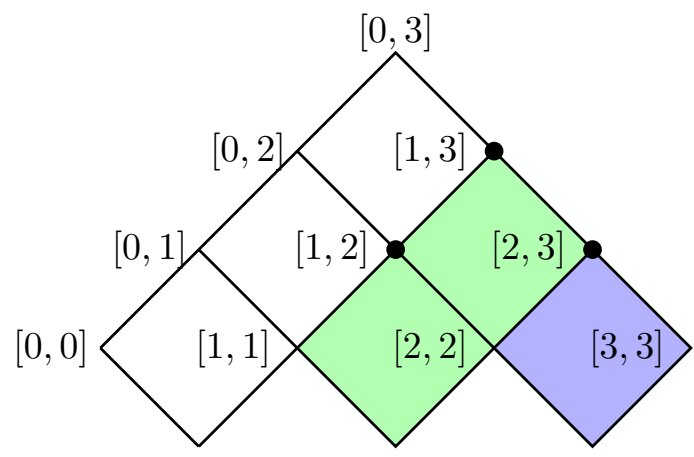

after setting $C$ 's in green and purple diamonds to be zero, three facets (black points) vanish and building set after deletion is

$$
\{[0,3],[0,2],[0,1],[0,0],[1,1],[2,2],[3,3]\} .
$$

One can further check that it's a cube (or $\mathscr{A}_{1}^{3}$ ).

We can play the game for any degenerations of $\mathscr{A}_{n}$, count black points and deleted $C$ 's in each degeneration, and find that for $\mathscr{A}_{2}, \mathscr{A}_{3}$ and $\mathscr{A}_{4}$, there're respectively 4,41 and 580 possible degenerations whose big polyhedra are simplices. For these degenerations, we can easily find their $u$ variables if the set of all vanishing facets are known.

Suppose $\mathscr{D}$ is the set of all vanishing facets of a degeneration in the building set, we can read from the mesh that

$$
F_{I^{\prime}}=\sum_{I \notin \mathscr{D}} M_{I^{\prime}}^{I} F_{I}
$$

for any $I^{\prime} \in \mathscr{D}$, where $M$ is a matrix with non-negative entries. Therefore,

$$
\prod_{I \in \mathscr{A} n} u_{I}^{F_{I}}=\prod_{I^{\prime} \in \mathscr{D}} u_{I^{\prime}}^{F_{I^{\prime}}} \cdot \prod_{I \notin \mathscr{D}} u_{I}^{F_{I}}=\prod_{I \notin \mathscr{D}}\left(u_{I} \prod_{I^{\prime} \in \mathscr{D}} u_{I^{\prime}}^{M_{I^{\prime}}^{I}}\right)^{F_{I}},
$$

so the new $u$ variables of the degeneration are

$$
\tilde{u}_{I}=u_{I} \prod_{I^{\prime} \in \mathscr{D}} u_{I^{\prime}}^{M_{I^{\prime}}^{I}}
$$

for any $I \notin \mathscr{D}$.

The simplest degenerations of $\mathscr{A}_{n}$ are those with one $C_{[i, i+1]}$ vanishing, where we get

$$
F_{[i, i+1]}=F_{i}+F_{i+1},
$$

with $F_{i}=F_{[i, i]}$, and we find the $u$ variables become

$$
\tilde{u}_{i}=u_{i} u_{[i, i+1]}, \quad \tilde{u}_{i+1}=u_{i+1} u_{[i, i+1]}, \quad \tilde{u}_{I}=u_{I} \text { for the other } I \neq[i, i+1] .
$$

They satisfy the following $u$ equation

$$
1-\tilde{u}_{i}=\prod_{0 \leq a \leq i-1} \tilde{u}_{[a, i-1]} \prod_{i+1<b \leq n} \tilde{u}_{[i+1, b]}, \quad 1-\tilde{u}_{i+1}=\prod_{0 \leq a<i-1} \tilde{u}_{[a, i]} \prod_{i+2 \leq b \leq n} \tilde{u}_{[i+2, b]},
$$


and the other $u$ equations are original $u$ equations for $\left\{u_{[i, j]}:[i, j] \neq[i, i],[i, i+1],[i+\right.$ $1, i+1]\}$ in terms of new variables. Similarly, one can easily find the $u$ variables and $u$ equations for the degenerations with $C_{[i, i+1]}=0$ for several $i$.

The next simplest degenerations of $\mathscr{A}_{n}$ may be products $\mathscr{A}_{i} \times \mathscr{A}_{n-i}$, which can be realized as the following mesh,

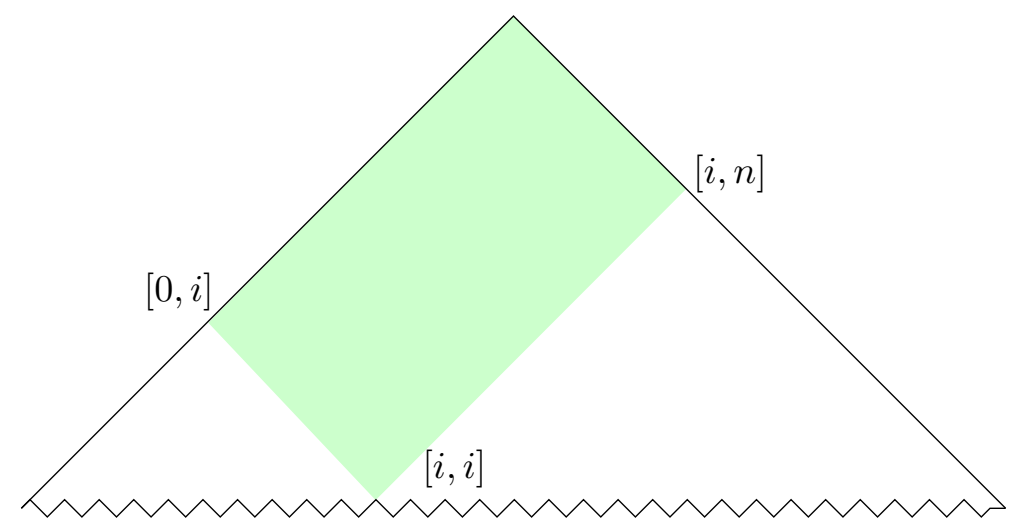

where $C$ 's in green region vanish, i.e. $C_{[a, b]}=0$ for $0 \leq a<i<b \leq n$. Vanishing facets of this degeneration are

$$
F_{[a, b]}=F_{[a, n]}+F_{[0, b]} \text { for } 0<a \leq i \leq b<n
$$

so new $u$ variables are

$$
\tilde{u}_{[0, b]}=u_{[0, b]} \prod_{a=1}^{i} u_{[a, b]}, \quad \tilde{u}_{[a, n]}=u_{[a, n]} \prod_{b=i}^{n-1} u_{[a, b]},
$$

and $\tilde{u}_{I}=u_{I}$ for the other $I$. The $u$ equations are the union of $u$ equations of the following two independent meshes

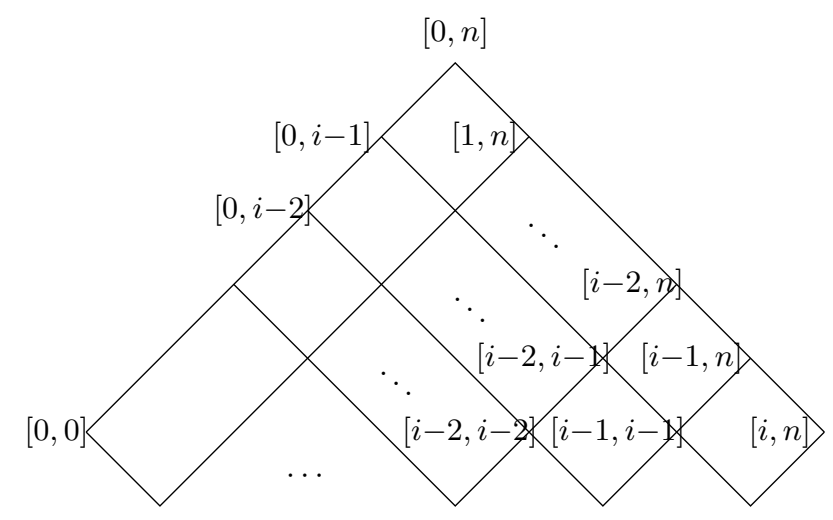




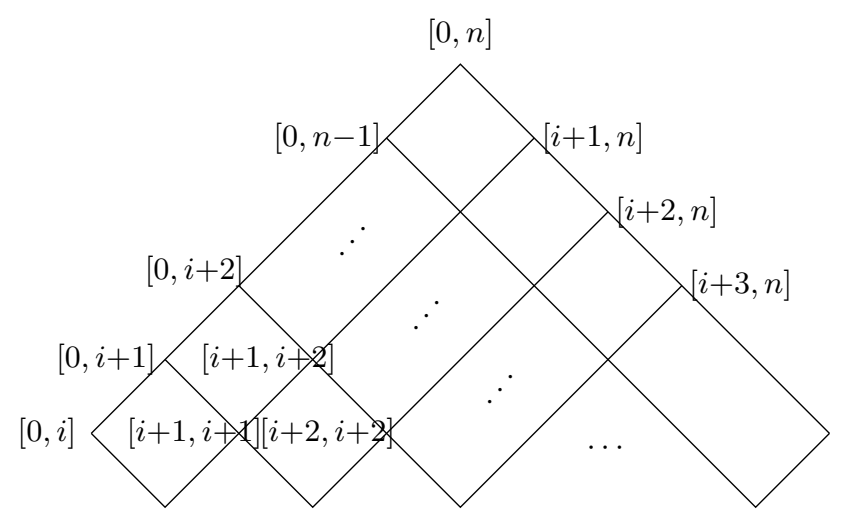

One could use this construction of products to produce any finite product $\mathscr{A}_{i_{1}} \times \cdots \times \mathscr{A}_{i_{k}}$. The direct proof of this argument for $\mathscr{A}_{n}$ is using extended $u$ equations $[5,15]$ of $\mathscr{A}_{n}$.

We can generalize the simplest cases in another way:

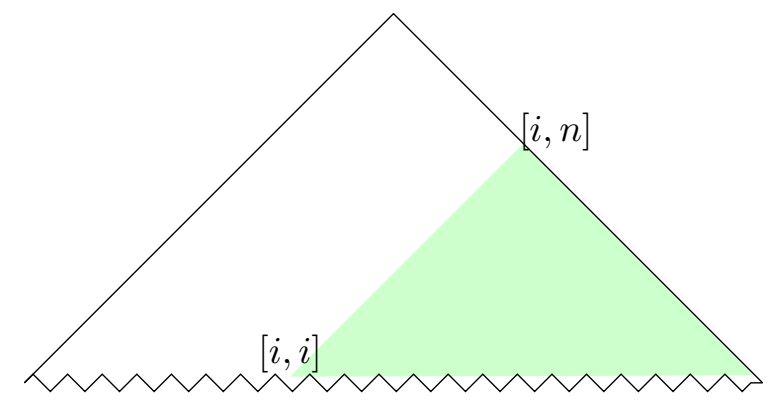

setting

$$
C_{[k, l]}=0 \quad \text { for } 0<i \leq k<l \leq n .
$$

In this case,

$$
F_{[k, l]}=\sum_{m=k}^{l} F_{[m, m]}
$$

for $i \leq k<l \leq n$. Therefore,

$$
\tilde{u}_{[k, k]}=\prod_{i \leq p \leq k \leq q \leq n} u_{[p, q]}=\frac{x_{k}}{x_{[i-1, k]}} \quad \text { for } k \in[i, n], \quad \tilde{u}_{I}=u_{I} \quad \text { for the other } I,
$$

and their $u$ equations are

$$
1-\tilde{u}_{[k, k]}=\prod_{j=0}^{i-1} \tilde{u}_{[j, k-1]}
$$

and the other $u$ equations are old $u$ equations in terms of new variables. When $i=1$, the degeneration is $\mathscr{A}_{1}^{n}$. 
Similarly, we can also consider the following degenerations:

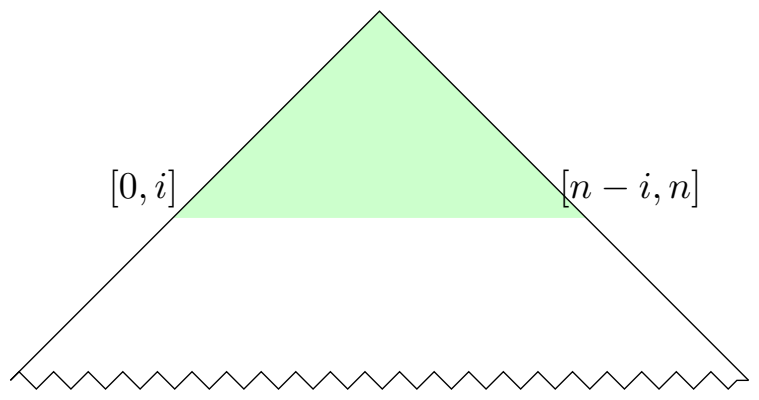

setting

$$
C_{[k, l]}=0 \quad \text { for } l-k>i \text {. }
$$

In this case,

$$
F_{[k, l]}=F_{[0, l]}+F_{[k, n]} \quad \text { for } l-k \geq i, 1 \leq k \leq n-i-1 \text { and } i+1 \leq l \leq n-1,
$$

then

$$
\tilde{u}_{[0, l]}=\prod_{j=0}^{l-i+1} u_{[j, l]}, \quad \tilde{u}_{[k, n]}=\prod_{j=k+i-1}^{n} u_{[k, j]} .
$$

Their $u$ equations are

$$
1-\tilde{u}_{[0, l]}=\prod_{a=l-i+2}^{l+1} \tilde{u}_{[a, n]} \cdot \prod_{a=l-i+2}^{l+1} \prod_{b=l+1}^{a+i-2} \tilde{u}_{[a, b]}, \quad 1-\tilde{u}_{[k, n]}=\prod_{b=k-1}^{k+i-2} \tilde{u}_{[0, b]} \cdot \prod_{b=k-1}^{k+i-2} \prod_{a=\max (b-i+2,1)}^{k-1} \tilde{u}_{[a, b]} .
$$

When $i=1$, we get $\mathscr{A}_{1}^{n}$ again.

\subsection{Degenerations of $\mathscr{B}_{n}$ and products}

\subsubsection{Degenerations of $\mathscr{B}_{n}$}

The general treatment of degenerations of $\mathscr{B}_{n}$ is more difficult than $\mathscr{A}_{n}$ because it has a long ABHY condition eq. (2.6). Here we only show some examples of degenerations of $\mathscr{B}_{n}$ with perfect $u$ equations.

The first degeneration of $\mathscr{B}_{n}$ with perfect $u$ equations is $\mathscr{A}_{n}$ because the building set of $\mathscr{A}_{n}$ is contained in the building set of $\mathscr{B}_{n}$.

The simplest degenerations of $\mathscr{B}_{n}$ are those with $C_{[i, i+1]}=0$. For which we get,

$$
F_{[i, i+1]}=F_{i}+F_{i+1},
$$

where $F_{i}=F_{[i, i]}$, so

$$
\tilde{u}_{i}=u_{i} u_{[i, i+1]}, \quad \tilde{u}_{i+1}=u_{i+1} u_{[i, i+1]}, \quad \tilde{u}_{I}=u_{I} \text { for the other } I \neq[i, i+1] .
$$

The $u$ equations of this degeneration are

$$
1-\tilde{u}_{i}=\tilde{u}_{[i+1, i-1]}^{2} \tilde{u}_{[i-1, i-1]} \prod_{a=i+2}^{i-2} \tilde{u}_{[a, i-1]} \tilde{u}_{[i+1, a]}, \quad 1-\tilde{u}_{i+1}=\tilde{u}_{[i+2, i]}^{2} \tilde{u}_{[i+2, i+2]} \prod_{a=i+3}^{i-1} \tilde{u}_{[a, i]} \tilde{u}_{[i+2, a]},
$$

and the other $u$ equations are old $u$ equations in terms of new $u$ variables. 
The above degeneration corresponds to deleting an element in $\mathscr{B}_{n}$, and the following degeneration corresponds to adding an element to $\mathscr{A}_{n}$.

Consider the building set obtained by adding the set $K_{i}=[0, i] \cup[i+2, n]$ to $\mathscr{A}_{n}$. It is also a degeneration of $\mathscr{B}_{n}$ by setting the other $C$ 's to be zero. It's easy to solve equations of the facets and get that

$C_{K_{i}}=F_{[0, i]}+F_{[i+2, n]}-F_{K_{i}}, \quad C_{[0, n]}=F_{K_{i}}+F_{[0, n-1]}+F_{[1, n-1]}-F_{[1, n-1]}-F_{[0, i]}-F_{[i+2, n]}$,

the other $C_{I}$ are given by eq. (2.3). Therefore, new $u$ variables are

$$
\tilde{u}_{K_{i}}=\frac{x_{K_{i}}}{x_{[0, n]}}, \quad \tilde{u}_{[0, i]}=\frac{x_{[0, n]}}{x_{K_{i}}} u_{[0, i]}, \quad \tilde{u}_{[i+2, n]}=\frac{x_{[0, n]}}{x_{K_{i}}} u_{[i+2, n]}
$$

and the other $\tilde{u}_{I}$ is equal to $u_{I}$. The $u$ equations are

$$
1-\tilde{u}_{K_{i}}=\prod_{I \ni i+1} \tilde{u}_{I}, \quad 1-\tilde{u}_{[0, i]}=\prod_{J} \tilde{u}_{J}^{J \|[0, i]} \prod_{K \supset[i+2, n]} \tilde{u}_{K}, \quad 1-\tilde{u}_{[i+2, n]}=\prod_{J} \tilde{u}_{J}^{J \|[i+2, n]} \prod_{K \supset[0, i]} \tilde{u}_{K},
$$

where $I \| J$ are compatability degree of $I$ and $J$ in $\mathscr{A}_{n}$, and the other $u$ equations are corresponding $u$ equations for $\mathscr{A}_{n}$ in new variables.

For example, adding $K_{0}$ to $\mathscr{A}_{2}$ we get $\mathscr{B}_{2}$, and adding $K_{0}$ to $\mathscr{A}_{3}$ gives the following $u$ equations

$$
\begin{array}{rlrl}
1-\tilde{u}_{3} & =\tilde{u}_{2} \tilde{u}_{12} \tilde{u}_{012} & 1-\tilde{u}_{01} & =\tilde{u}_{2} \tilde{u}_{12} \tilde{u}_{23} \tilde{u}_{023} \tilde{u}_{123} \\
1-\tilde{u}_{1} & =\tilde{u}_{0} \tilde{u}_{2} \tilde{u}_{23} \tilde{u}_{023}^{2} & 1-\tilde{u}_{2} & =\tilde{u}_{1} \tilde{u}_{3} \tilde{u}_{01} \\
1-\tilde{u}_{012} & =\tilde{u}_{3} \tilde{u}_{23} \tilde{u}_{023} \tilde{u}_{123} & 1-\tilde{u}_{023} & =\tilde{u}_{1} \tilde{u}_{01} \tilde{u}_{12} \tilde{u}_{012} \tilde{u}_{123} \\
1-\tilde{u}_{123} & =\tilde{u}_{0} \tilde{u}_{01} \tilde{u}_{012} \tilde{u}_{023} & 1-\tilde{u}_{0} & =\tilde{u}_{1} \tilde{u}_{12} \tilde{u}_{23} \tilde{u}_{123}^{2} \\
1-\tilde{u}_{12} & =\tilde{u}_{0} \tilde{u}_{3} \tilde{u}_{01} \tilde{u}_{23} \tilde{u}_{023}^{2} & 1-\tilde{u}_{23} & =\tilde{u}_{0} \tilde{u}_{1} \tilde{u}_{01}^{2} \tilde{u}_{12} \tilde{u}_{012}^{2} .
\end{array}
$$

More generally we can consider adding a triangle namely $\cup_{l=0}^{k-1} \cup_{m=l}^{k-1} K_{i, l, m}$ with $K_{i, l, m}=$ $\{[0, i+l] \cup[i+2+m, n]\}$ and $k=1, \ldots,(n-1)$ to the building set of $\mathscr{A}_{n}$. For $k=1$ we get the previous example and for $k=n-1$ we get $\mathscr{B}_{n}$.

Without loss of generality we shall consider only the $i=0$ case and we shall denote $K_{0, l, m}$ by just $K_{l, m}$. The $u$ variables are

$$
\tilde{u}_{K_{l, m}}=\left\{\begin{array}{l}
\frac{x_{K_{l, m}}}{x_{[0, n]}}, \quad \text { if } m=l \\
\frac{x_{K_{l, m}} x_{K_{l+1, m-1}}}{x_{K_{l+1, m}} x_{l, m-1}}, m \neq l
\end{array} \quad \tilde{u}_{[0, l]}=\frac{u_{[0, l]}}{\prod_{j=0}^{k-l-1} \tilde{u}_{K_{l, l+j}}}, \quad \tilde{u}_{[m+2, n]}=\frac{u_{[m+2, n]}}{\prod_{j=0}^{m} \tilde{u}_{K_{j, m}}}\right.
$$

with the understanding that $K_{1,0}=K_{2,0}=[0, n]$ and the other $\tilde{u}_{I}$ is equal to $u_{I}$. 
The $u$ equations are:

$$
\begin{aligned}
1-\tilde{u}_{[0, l]}= & \left(\prod_{i=0}^{l-1} \prod_{j=i+1}^{k+1} \tilde{u}_{[0, i] \cup[j, n]} \prod_{j=i+1}^{l+1} \tilde{u}_{[0, i] \cup[j, n]}\right)\left(\prod_{i=1}^{l+1} \prod_{j=l+1}^{n} \tilde{u}_{[i, j]}\right)\left(\prod_{i=1}^{k+1} \tilde{u}_{[i, n]}\right), \\
1-\tilde{u}_{[m+2, n]}= & \left(\prod_{j=0}^{m} \tilde{u}_{[0, j]} \prod_{i=1}^{n-m-1} \tilde{u}_{[0, m+i]}^{2}\right)\left(\prod_{i=m+1}^{n-1} \prod_{j=1}^{m+1} \tilde{u}_{[j, i]}\right) \\
& \times\left(\prod_{j=0}^{k-m-2} \prod_{i=1}^{m} \tilde{u}_{[0, i] \cup[3+j+m, n]} \prod_{i=m+1}^{j+m+1} \tilde{u}_{[0, i] \cup[3+j+m, n]}^{2}\right) \\
1-\tilde{u}_{K_{l, m}}= & \left(\prod_{a=0}^{1} \prod_{i=l+1}^{n} \tilde{u}_{[a, i]}\right)\left(\begin{array}{l}
\prod_{K_{l^{\prime}, m^{\prime}} \not K_{l, m}} \tilde{u}_{K_{l, m}, K^{\prime}, m^{\prime}}^{\left(l^{\prime}, m^{\prime}\right) \mid(l, m)} \\
l_{l^{\prime}, m^{\prime}}
\end{array}\right)\left(\prod_{j=2}^{m+1} \prod_{i=l+1}^{n} \tilde{u}_{[j, i]}\right) \\
& \times\left\{\begin{array}{l}
1, \text { if } l=m \\
\prod_{i=0}^{l+1} \prod_{j=m+1}^{n} \tilde{u}_{[i, j]}, \text { if } l \neq m
\end{array}\right.
\end{aligned}
$$

where $\left(l^{\prime}, m^{\prime}\right) \|(l, m)$ is the compatability degree of $K_{l, m}$ with respect to $K_{l^{\prime}, m^{\prime}}$ in $\mathscr{B}_{n}$ with the understanding that $\tilde{u}_{[0, n]}=1$ and the other $u$ equations are corresponding $u$ equations for $\mathscr{A}_{n}$ in new variables.

\subsubsection{Products of $\mathscr{A}$ and $\mathscr{B}$}

We can also construct binary geometries of the type $\prod_{i=1}^{n} X_{i}$ where $X_{i}=\mathscr{A}_{i}$ or $\mathscr{B}_{i}$ which are not necessarily degenerations of $\mathscr{A}$ or $\mathscr{B}$. Without loss of generality we consider only the case $\mathscr{A}_{n} \times \mathscr{B}_{m}$. We can do this in a couple of simple ways as follows:

(1) We consider the building set $\mathbf{B}_{1} \cup \mathbf{B}_{2}$ with $\mathbf{B}_{1}=\{[i, j]: 0 \leq i \leq j \leq n\}$ and $\mathbf{B}_{2}=\{[i, i+k]: n \leq i, k \leq m+n\}$. Notice that these are just the building sets of $\mathscr{A}_{n}$ and $\mathscr{B}_{m}$ respectively.

If $\mathbf{B}_{1}$ and $\mathbf{B}_{2}$ were disjoint then it's obvious that the stringy integral $\mathcal{I}_{\mathbf{B}}$ in (1.2) directly factorizes into $\mathcal{I}_{\mathbf{B}_{1}} \times \mathcal{I}_{\mathbf{B}_{2}}$, however the singlet $\{n\}$ belongs to both $B_{1}$ and $B_{2}$. But by rescaling the variables in $B_{2}$ by $x_{i} \rightarrow x_{n} x_{i}$ for all $i=n+1, \ldots, n+m$ it is straightforward to see that the $\mathcal{I}_{\mathbf{B}}$ does indeed factorize into $\mathcal{I}_{\mathbf{B}_{1}} \times \mathcal{I}_{\mathbf{B}_{2}}$ with $x_{n}$ playing the role of $x_{0}=1$ in $B_{2}$.

Notice that the above proof did not use the fact that $\mathbf{B}_{1}, \mathbf{B}_{2}$ were building sets of $\mathscr{A}, \mathscr{B}$ and can easily be carried forward to obtain any product of generalized permutohedra. The interested reader may refer appendix (A) for details.

(2) Consider the building set $\mathbf{B}_{1} \cup \mathbf{B}_{2}$ with $\mathbf{B}_{1}=\{[i, i+k]: 0 \leq i, k \leq m\}$ and $\mathbf{B}_{2}=$ $\{[i, j]: m<i<j \leq n+m\} \cup\{[0, i]: m \leq i \leq n+m\}$. $\mathbf{B}_{1}$ is obviously the building set for $\mathscr{B}_{m}$, while $\mathbf{B}_{2}$ can be viewed as the building set for $\mathscr{A}_{n}$ by identifying $[0, n]$ as a singleton in $\mathbf{B}_{2}$. Due to this identification, one can easily write down the $u$ variables separately for $\mathbf{B}_{1}$ and $\mathbf{B}_{2}$, and check that these $u$ variables satisfy the $u$ equations for $\mathscr{A}_{n} \times \mathscr{B}_{m}$. 


\section{Stringy canonical forms and binary geometries for generalized permu- tohedra}

In this section we shall argue that a large class of generalized permutohedra which are realised as degenerations of permutohedron $\mathscr{P}_{n}$ are binary geometries. Before we proceed, we shall review some details about the generalized permutohedra [21, 24] which we shall use throughout the paper.

\subsection{Generalized permutohedra}

A generalized permutohedron is a polytope that can be obtained as Minkowski sums and differences of coordinate simplices.

Let $\Delta_{[0, n]}=$ ConvexHull $\left(e_{1}, \ldots, e_{n}\right)$ be the standard coordinate simplex in $\mathbb{R}^{n+1}$. Then we have:

$$
\mathscr{P}_{n}\left(\left\{y_{I}\right\}\right)=\sum y_{I} \cdot \Delta_{I}
$$

for some collection of subsets $I \in[0, n]$. We shall restrict ourselves to the large class of generalized permutohedra which admit such a realisation only as Minkowski sums except translations i.e, $y_{I} \geq 0$ for all non-singlets $I^{5}$ which are called nestohedra. Throughout the paper we shall mean nestohedra or products of nestohedra whenever we refer to generalized permutohedra.

The combinatorial structure of the generalized permutohedron $\mathscr{P}_{n}\left(\left\{y_{I}\right\}\right)$ (nestohedra) is independent of $y_{I}$ and depends only on the collection of subsets $I \in[0, n]$ of the building set $\mathbf{B}$, which is a collection of non-empty subsets of $[0, n]$ satisfying the following:

(1) If $I, J \in \mathbf{B}$ and $I \cap J \neq \varnothing$, then $I \cup J \in \mathbf{B}$.

(2) $\mathbf{B}$ contains all singletons $\{i\}$ for $i \in S$.

Here are some interesting examples of generalized permutohedra:

- If $\mathbf{B}=\{[i] \mid i=0, \ldots, n\}$ is the complete flag of intervals, then $\mathscr{P}_{n}\left(\left\{y_{I}\right\}\right)$ is the Stanley-Pitman polytope or Hypercube.

- If $\mathbf{B}$ corresponds to all the non empty subsets of $[0, n]$ and $Y_{I}=y_{|I|}$ i.e., the variables $Y_{I}$ are equal for all subsets of the same cardinality, then $\mathscr{P}_{n}\left(\left\{y_{I}\right\}\right)$ is the usual permutohedron $\mathscr{P}_{n}$.

- If $\mathbf{B}=\{[i, j] \mid 0 \leq i \leq j \leq n\}$ is the set of consecutive intervals, then $\mathscr{P}_{n}\left(\left\{y_{I}\right\}\right)$ is the associahedron $\mathscr{A}_{n}$.

- If $\mathbf{B}=\{[i, i+k]: 0 \leq i, k \leq n\}$ is the set of cyclic intervals, then $\mathscr{P}_{n}\left(\left\{y_{I}\right\}\right)$ is the cyclohedron $\mathscr{B}_{n}$.

- Let $\Gamma$ be a graph on the vertex set $[0, n]$. Let us assume that $\mathbf{B}=\mathbf{B}(\Gamma)$ is the set of subsets $I \in[0, n]$ such that the induced graph $\Gamma$ is connected, then $\mathscr{P}_{n}\left(\left\{y_{I}\right\}\right)$ is a graph associahedron.

\footnotetext{
${ }^{5}$ This is mainly due to the subtle nature of the Minkowski difference operation, which makes the shape of the resulting polytope dependent on the relative magnitudes of $y_{I}$ 's. We allow $y_{I}$ for singlets to be negative as it is only a translation and to connect with (1.7).
} 
Nested complex. To describe the combinatorial structure we need the notion of nested complex. We shall only summarize the necessary results here and let the reader interested in the details to refer [21, 24]. A subset $\mathbf{N}$ in the building set $\mathbf{B}$ is called a nested set if it satisfies the following conditions:

(1) For any $I, J \in \mathbf{N}$, we either have $I \subset J$ or $J \subset I$ or $I$ and $J$ are disjoint.

(2) For any collection of $k \geq 2$ disjoint subsets $J_{1}, J_{2}, \ldots, J_{k} \in \mathbf{N}$ their union $J_{1} \cup \cdots \cup J_{k}$ is not in $\mathbf{B}$.

(3) $\mathbf{N}$ contains all maximal elements of $\mathbf{B}$.

The nested complex $\mathbf{N}(\mathbf{B})$ is defined as the poset of the set of all nested sets in $\mathbf{B}$ ordered by inclusion.

Facial structure. Let us assume that the set B associated with a generalized permutohedron $\mathscr{P}_{n}\left(\left\{y_{I}\right\}\right)$ is a building set on $[0, n]$. Then the poset of faces $\mathscr{P}_{n}\left(\left\{y_{I}\right\}\right)$ ordered by reverse inclusion is isomorphic to the nested complex $\mathbf{N}(\mathbf{B})$.

In particular, it means that (1) the set of facets of the generalized permutohedron just corresponds to the set of all elements in $\mathbf{B}$ excluding the maximal element, and (2) two facets corresponding to the sets $I$ and $J$ in $\mathbf{B}$ intersect i.e., are compatible with each other, if and only if either $I \subset J, J \subset I$ or $I \cap J=\varnothing$ and $I \cup J \notin \mathbf{B}$.

The face $P_{\mathbf{N}}$ of $\mathscr{P}_{n}\left(\left\{y_{I}\right\}\right)$ associated with the nested set $\mathbf{N} \in \mathbf{N}(\mathbf{B})$ is given by

$$
P_{\mathbf{N}}=\left\{\left(t_{0}, \ldots, t_{n}\right) \in \mathbb{R}^{n+1} \mid \sum_{i \in I} t_{i}=z_{I} \text { for } I \in \mathbf{N} ; \sum_{i \in J} t_{i} \geq z_{J}, \text { for } J \in \mathbf{B}\right\},
$$

where $z_{I}=\sum_{J \subset I, J \in \mathbf{B}} y_{J}$ for $I \in \mathbf{B}$ and coordinate simplices $\left\{\Delta_{I}\right\}_{I \in \mathbf{B}}$ are living in $\left\{t_{i}\right\}_{0 \leq i \leq n}$-space. In particular, the equation of the facet labeled by nested set $\{[0, n], I\}$ of $\mathscr{P}_{n}\left(\left\{y_{I}\right\}\right)$ is given by

$$
\sum_{i \in I} t_{i}=\sum_{J \subset I, J \in \mathbf{B}} y_{J}
$$

Another description of the faces is the following: for each decomposition $[0, n]=$ $\sqcup_{I \subset \mathbf{N}} S_{I}$ where $S_{I}$ are non-empty, the face $P_{\mathbf{N}}$ of $\mathscr{P}_{n}\left(\left\{y_{I}\right\}\right)$ associated with the nested set $\mathbf{N} \in \mathbf{N}(\mathbf{B})$ is

$$
P_{\mathbf{N}}=\sum_{\substack{J \subset \mathbf{B} \\ J \cap S_{I} \neq \varnothing}} y_{J} \Delta_{J \cap S_{I}}
$$

In particular each facet of $\mathscr{P}_{n}(\{y\})$ corresponding to a set $I \in \mathbf{B}$ is isomorphic to the product $\mathscr{P}_{\mathbf{B}_{I}} \times \mathscr{P}_{\mathbf{B}_{\bar{I}}}$ of generalized permutohedra corresponding to the building sets

$$
\mathbf{B}_{I}=\{J \mid J \subset I, J \in \mathbf{B}\}
$$

and

$$
\mathbf{B}_{\bar{I}}=\{J-I \mid J \in \mathbf{B}\}
$$

Let us look at a couple of examples to appreciate these facts better:

(1) Consider the $2 \mathrm{~d}$ associahedron with building set

$$
\mathbf{B}=\{\{0\},\{1\},\{2\},\{0,1\},\{1,2\},\{0,1,2\}\} .
$$




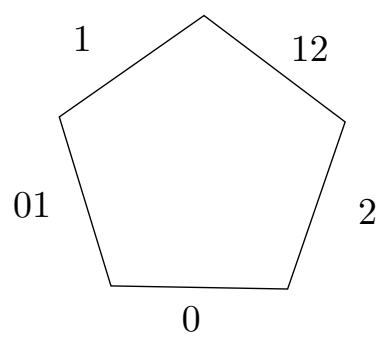

(a)

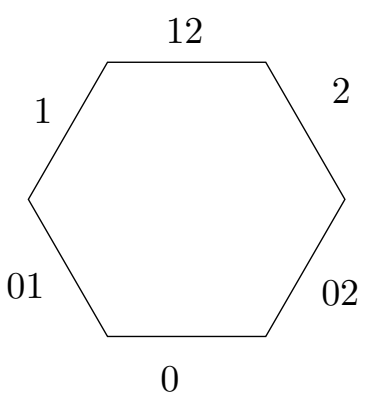

(b)

Figure 1. Facets of the 2d associahedron (a) and permutohedron (b) as generalized permutohedra.

This polygon has 5 facets which correspond to the sets $\{\{0\},\{1\},\{2\},\{0,1\},\{1,2\}\}$ and their compatability is shown in the figure below.

(2) Consider the 2d cyclohedron/permutohedron with building set

$$
\mathbf{B}=\{\{0\},\{1\},\{2\},\{0,1\},\{1,2\},\{0,2\},\{0,1,2\}\} .
$$

This polygon has 6 facets which correspond to the sets $\{\{0\},\{1\},\{2\},\{0,1\},\{1,2\},\{0,2\}\}$ and their compatability is shown in the figure below.

The big polyhedron of generalized permutohedron is a simplex. Thus it's quite natural to wonder if the associated $u$ variables satisfy some kind of $u$ equations. We claim and we shall show in the next section that this is indeed the case. Further we show that all these polytopes are binary geometries even though some of them do not admit perfect $u$ equations.

\subsection{ABHY-like realizations and $u$ variables for generalized permutohedra}

As mentioned earlier, one can write down a natural stringy integral (1.7) for generalized permutahedron $\mathscr{P}(\mathbf{B})$, and its polytope is given by the Minkowski sum

$$
\mathscr{P}_{n}\left(\left\{-S_{I}|I \in \mathbf{B},| I \mid>1\right\}\right)=-\sum_{I \in \mathbf{B},|I|>1} S_{I} \cdot \Delta_{I},
$$

where $\Delta_{I}=$ ConvexHull $\left(\left\{e_{i}\right\}_{i \in I}\right)$ is the coordinate simplex in $\left(S_{0}, \ldots, S_{n}\right)$-space, so the equation of the facet labeled by $I$ is given by eq. (3.3):

$$
F_{I}:=\sum_{J \subset I, J \in \mathbf{B}} S_{J}=0 .
$$

From the previous subsection we know that the big polyhedron of a generalized permutohedron is a simplex. Therefore, we can write down the matrix $W_{A}^{J}$ of the facets and obtain

$$
S_{J}=F_{A}\left(W^{-1}\right)_{J}^{A}
$$


and its $u$ variables

$$
u_{A}=\prod_{J} x_{J}^{\left(W^{-1}\right)_{J}^{A}}
$$

Within generalized permutohedra, the original permutohedron is quite simple and important because any other generalized permutohedron can be obtained as a degeneration of the permutohedron. The building set of the $n$-dimensional permutohedron $\mathscr{P}_{n}$ is the set of all non-empty subsets of $[0, n]$. To get $u$ equations, we should solve for $S_{J}$ from $F_{J}$ in eq. (3.7), the solution for the permutohedron $\mathscr{P}_{n}$ is

$$
S_{I}=\sum_{J \subset I}(-1)^{|J|-|I|} F_{J},
$$

This gives us the following ABHY polytope: setting $S_{I}=-C_{I}$ for any non-singleton $I$, where $C_{I}$ are positive constants, and the polytope is given by the alternating sum

$$
F_{I} \geq 0, \quad-C_{I}=\sum_{J \neq \varnothing, J \subset I}(-1)^{|J|-|I|} F_{J}
$$

in the $\left\{F_{I}\right\}$-space. By eq. (3.2), this polytope is the $n$-dimensional permutohedron $\mathscr{P}_{n}\left(\left\{C_{I}\right\}\right)$, and it is nice that it admits a simple ABHY-like realization. The ABHY construction of any generalized permutohedron can be obtained by setting $C_{I}=0$ in $\mathscr{P}_{n}\left(\left\{C_{I}\right\}\right)$ for all $I$ not in its building set.

For example, the building set of $\mathscr{A}_{2}$ is $\mathbf{B}\left(\mathscr{P}_{2}\right)-\{\{0,2\}\}$, its ABHY construction can be obtained from $\mathscr{P}_{2}$ by setting $C_{02}=0$,

$$
0=-C_{02}=F_{02}-F_{2},
$$

therefore the whole ABHY conditions for $\mathscr{A}_{2}$ is

$$
-C_{01}=F_{01}-F_{0}-F_{1}, \quad-C_{12}=F_{12}-F_{1}-F_{2}, \quad-C_{012}=-F_{01}-F_{12}+F_{1}, \quad F_{I} \geq 0,
$$

and its solution in $\left(F_{1}, F_{2}\right)$-space is

$$
\left\{\begin{array}{l}
F_{1} \geq 0, F_{2} \geq 0 \\
F_{0}=C_{01}+C_{12}+C_{012}-F_{1}-F_{2} \geq 0 \\
F_{12}=F_{1}+F_{2}-C_{12} \geq 0 \\
F_{01}=C_{012}+C_{12}-F_{2} \geq 0
\end{array}\right.
$$

For another example, consider the building set

$$
\mathbf{B}=\{\{0\},\{1\},\{2\},\{3\},\{0,1,2\},\{1,3\},\{0,1,2,3\}\} .
$$

It's a degeneration of $\mathscr{P}_{3}$, where

$$
C_{01}, C_{02}, C_{03}, C_{12}, C_{23}, C_{013}, C_{023}, C_{123}=0
$$


and its $u$ variables and $u$ equations can be found at the end of section (3.4). By solving the ABHY conditions for these $C_{I}=0$, we get the vanishing facets

$$
\begin{aligned}
& F_{0 i}=F_{0}+F_{i}, \quad F_{12}=F_{1}+F_{2}, \quad F_{23}=F_{2}+F 3, \\
& F_{013}=F_{13}+F_{0}, \quad F_{023}=F_{0}+F_{2}+F_{3}, \quad F_{123}=F_{2}+F_{13} \text {, }
\end{aligned}
$$

and the remaining $\mathrm{ABHY}$ conditions

$$
C_{13}=F_{1}+F_{3}-F_{13}, \quad C_{012}=F_{0}+F_{1}+F_{2}-F_{012}, \quad C_{0123}=F_{012}+F_{13}-F_{1},
$$

its solution in $\left(F_{1}, F_{2}, F_{3}\right)$-space is

$$
\left\{\begin{aligned}
F_{1} & \geq 0, F_{2} \geq 0, F_{3} \geq 0 \\
F_{0} & =C_{012}+C_{0123}+C_{13}-F_{1}-F_{2}-F_{3} \geq 0 \\
F_{13} & =F_{1}+F_{3}-C_{13} \geq 0 \\
F_{012} & =C_{0123}+C_{13}-F_{3} \geq 0 .
\end{aligned}\right.
$$

Thanks to eq. (3.8), using the picture of big polyhedron, it gives $u$ variables of permutohedron $\mathscr{P}_{n}$

$$
u_{I}=\prod_{J \supset I} x_{J}^{(-1)^{|I|-|J|}}
$$

since

$$
\prod_{I \subset[0, n]} u_{I}^{F_{I}}=\prod_{I \subset[0, n]} x_{I}^{S_{I}}=\prod_{J \subset I \subset[0, n]} x_{I}^{(-1)^{|J|-|I|} F_{J}}=\prod_{J \subset[0, n]} \prod_{I \supset J}\left(x_{I}^{(-1)^{|J|-|I|}}\right)^{F_{J}} .
$$

Let us look at the $n=2,3$ examples:

2d permutohedron. In this case, the $u$ variables can be written in terms of $x$ variables as:

$$
u_{0}=\frac{x_{0} x_{012}}{x_{01} x_{02}}, \quad u_{1}=\frac{x_{1} x_{012}}{x_{01} x_{12}}, \quad u_{2}=\frac{x_{2} x_{012}}{x_{02} x_{12}}, \quad u_{01}=\frac{x_{01}}{x_{012}}, \quad u_{02}=\frac{x_{02}}{x_{012}}, \quad u_{12}=\frac{x_{12}}{x_{012}} .
$$

The $u$ equations are:

$$
\begin{aligned}
1-u_{0} & =u_{1} u_{2} u_{12}^{2}, 1-u_{1}=u_{0} u_{2} u_{02}^{2}, 1-u_{2}=u_{0} u_{1} u_{01}^{2} \\
1-u_{01} & =u_{2} u_{02} u_{12}, 1-u_{02}=u_{1} u_{01} u_{12}, 1-u_{12}=u_{0} u_{01} u_{02} .
\end{aligned}
$$

which are the same as the $u$ equations for $\mathscr{B}_{2}$.

3d permutohedron. In this case, the $u$ variables can be written in terms of $x$ variables as:

$$
u_{0}=\frac{x_{0} x_{012} x_{013} x_{023}}{x_{01} x_{02} x_{03} x_{0123}}, \quad u_{01}=\frac{x_{01} x_{0123}}{x_{012} x_{013}}, \quad u_{02}=\frac{x_{02} x_{0123}}{x_{012} x_{023}}, \quad u_{012}=\frac{x_{012}}{x_{0123}},
$$


and their cyclic permutations. The $u$ equations are

$$
\begin{aligned}
1-u_{0} & =u_{1} u_{2} u_{3} u_{12}^{2} u_{23}^{2} u_{13}^{2} u_{123}^{3}\left(1+u_{0} u_{023} u_{013} u_{012} u_{03} u_{01} u_{02}\right) \\
1-u_{01} & =u_{2} u_{3} u_{23}^{2} u_{12} u_{13} u_{123}^{2} u_{023}^{2} u_{02} u_{03} \\
1-u_{02} & =u_{1} u_{3} u_{13}^{2} u_{12} u_{23} u_{123}^{2} u_{013}^{2} u_{01} u_{03} \\
1-u_{012} & =u_{3} u_{03} u_{13} u_{23} u_{013} u_{023} u_{123}
\end{aligned}
$$

and their cyclic permutations. The $u$ variables and $u$ equations of $\mathscr{P}_{4}$ can be found in appendix (B).

\subsection{Permutohedra as binary geometries}

In this subsection, we will show that the $u_{I}$ 's for the permutohedron $\mathscr{P}_{n}$, which are defined by eq. (3.10), have the desired binary property. Recall that $u_{I}$ and $u_{J}$ are compatible if and only if $I \subset J$ or $J \subset I$, otherwise they are incompatible. We will see that as $u_{I} \rightarrow 0$, all the incompatible $u_{J} \rightarrow 1$. Furthermore, we will show that the $u$ variables compatible with $u_{I}$ become the $u$ variables for the facet $\mathscr{P}_{|I|-1} \times \mathscr{P}_{n-|I|}$.

A crucial observation here is that $u_{I} \rightarrow 0$ is equivalent to $x_{I} \rightarrow 0$ according to (3.10) since all $x_{i}$ are positive. We can approach this limit by replacing $x_{i}$ with $\epsilon x_{i}$ for all $i \in I$ then taking $\epsilon \rightarrow 0$. Then the remaining task is just to consider the behaviour of the other $u_{J}$ 's under this limit.

Let us first show that the $u$ variables incompatible with $u_{I}$ become 1 as $u_{I}$ goes to 0 . It is obvious that $u_{I}$ and $u_{J}$ are incompatible if either (1) $I \cap J=\varnothing$ or (2) $I \cap J \neq \varnothing, I, J$. For the first case, let us denote $K=[0, n]-I-J$ as the complement set of $[0, n]$ with respect to $I \cup J$. Then we find the logarithm of $u_{J}$ can be written as

$$
\log u_{J}=\sum_{\kappa \subset K, \psi \subset I}(-1)^{|\kappa|+|\psi|} \log \left(x_{J}+x_{\kappa}+x_{\psi}\right) .
$$

As $x_{i} \rightarrow 0$ with $i \in I$, we have

$$
\log u_{J} \rightarrow \sum_{\kappa \subset K}(-1)^{|\kappa|} \log \left(x_{J}+x_{\kappa}\right)\left(\sum_{\psi \subset I}(-1)^{|\psi|}\left(\begin{array}{l}
|I| \\
|\psi|
\end{array}\right)\right)=0,
$$

where the binomial expansion of $(1-1)^{|I|}$ has been used. For the second case, the logarithm of $u_{J}$ can be written as

$$
\log u_{J}=\sum_{\psi^{\prime} \subset I^{\prime}, \kappa \subset K}(-1)^{\left|\psi^{\prime}\right|+|\kappa|} \log \left(x_{J^{\prime}}+x_{(I \cap J)}+x_{\psi^{\prime}}+x_{\kappa}\right),
$$

where $I^{\prime}=I-J, J^{\prime}=J-I$ and $K=[0, n]-(I \cup J)$. Then a similar argument as in (3.14) gives $\log u_{J} \rightarrow 0$ under the limit of all $x_{i} \rightarrow 0$ with $i \in I$.

Now let us consider the behaviour of compatible $u$ variables under this limit. For $J \subset I$, we again introduce $I^{\prime}=I-J$ and $K=[0, n]-I$, then the logarithm of $u_{J}$ can written as

$$
\log u_{J}=\sum_{\psi^{\prime} \subset I^{\prime}, \kappa \subset K}(-1)^{\left|\psi^{\prime}\right|+|\kappa|} \log \left(x_{J}+x_{\psi^{\prime}}+x_{\kappa}\right)
$$


Next, we replace $x_{i}$ with $\epsilon x_{i}$ for all $i \in I$ and take $\epsilon \rightarrow 0$, then

$$
\begin{aligned}
\log u_{J} & \rightarrow \lim _{\epsilon \rightarrow 0} \sum_{\psi^{\prime} \subset I^{\prime}, \kappa \subset K}(-1)^{\left|\psi^{\prime}\right|+|\kappa|} \log \left(\epsilon x_{J}+\epsilon x_{\psi^{\prime}}+x_{\kappa}\right) \\
& =\lim _{\epsilon \rightarrow 0}\left(\log \epsilon+\sum_{\kappa \subset K, \kappa \neq \varnothing} \log x_{\kappa}\right)\left(\sum_{\psi^{\prime} \subset I^{\prime}}(-1)^{\left|\psi^{\prime}\right|}\left(\begin{array}{c}
|I| \\
\left|\psi^{\prime}\right|
\end{array}\right)\right)+\sum_{\psi^{\prime} \subset I^{\prime}}(-1)^{\left|\psi^{\prime}\right|} \log \left(x_{J}+x_{\psi^{\prime}}\right) \\
& =\sum_{\psi^{\prime} \subset I^{\prime}}(-1)^{\left|\psi^{\prime}\right|} \log \left(x_{J}+x_{\psi^{\prime}}\right) .
\end{aligned}
$$

Obviously, the expression in the last line of (3.17) is a $u$ variable for the permutohedron $\mathscr{P}_{|I|-1}$. For $J \supset I$, under the limit of $x_{I} \rightarrow 0$, the logarithm of $u_{J}$ simply becomes

$$
\log u_{J}=\sum_{\kappa \subset K}(-1)^{\kappa} \log \left(x_{I}+x_{J-I}+x_{\kappa}\right) \rightarrow \sum_{\kappa \subset K}(-1)^{\kappa} \log \left(x_{J-I}+x_{\kappa}\right),
$$

where $K=[0, n]-J$, which is a $u$ variable for the permutohderon $\mathscr{P}_{n-|I|}$.

\subsubsection{The $u$ equations of $\mathscr{P}_{n}$}

For a permutohedron $\mathscr{P}_{n}$, we can directly use eq. (3.7) to express $x_{I}$ in terms of $\left\{u_{I}\right\}$ which gives us many algebraic equations for the $u$ variables:

$$
\prod_{I} x_{I}^{S_{I}}=\prod_{J} u_{J}^{F_{J}}=\prod_{J} \prod_{I \subset J} u_{J}^{S_{I}}=\prod_{I}\left(\prod_{J \supset I} u_{J}\right)^{S_{I}},
$$

so

$$
x_{I}=\prod_{J \supset I} u_{J}
$$

for any $I \in \mathscr{P}_{n}$, where we introduce a fake $u$ variable $u_{[0, n]}:=x_{[0, n]}$. We know some algebraic equations of $\left\{x_{I}\right\}$, for example, linear equations $x_{I}+x_{J}=x_{I \cup J}$ for $I \cap J=\varnothing$, so

$$
\prod_{K \supset I} u_{K}+\prod_{K \supset J} u_{K}=\prod_{K \supset I \cup J} u_{K}
$$

or equivalently

$$
\prod_{I \subset K, K \not \supset I \cup J} u_{K}+\prod_{J \subset K, K \not \supset I \cup J} u_{K}=1 .
$$

We can in fact use the above relations to find the $u$ equations for $\mathscr{P}_{n}$ recursively as follows:

for $I, J$ such that $|I|=n$ and $J=[0, n]-I=\{i\}$ we have $1-u_{I}=\prod_{i \in J} u_{J}$ which is already an $u$ equation for $u_{I}$. For $|I|=n-1, J=\{i\}$ and $\{k\}=[0, n]-(I \cup J)$ we have $1-u_{I} u_{I \cup\{k\}}=\prod_{\substack{\{i\} \in J \\ J \neq I \cup\{i\}}} u_{J}$ by rewriting $1-u_{I} u_{I \cup\{k\}}=1-u_{I}+u_{I}\left(1-u_{I \cup\{k\}}\right)$ and using the above equations we get

$$
1-u_{I}=\prod_{\substack{\{i\} \in J \\ J \neq I \cup\{i\}}} u_{J}-u_{I} \prod_{\{i\} \in K} u_{K}=\prod_{\substack{\{i\} \in J \\ J \neq I \cup\{i\}}} u_{J}\left(1-u_{I} u_{I \cup\{i\}}\right)
$$


More generally we can find the $u$ equation for any $I$ recursively by starting from $|I|=n$. We can argue this inductively by assuming we know the $u$ equations for all $I$ such that $|I| \geq k$.

We can now look at all the sets $\left\{I_{1}, \ldots, I_{n}\right\}$ that contain $|I|=k-1$ but not $I \cup J$ and arrange them as $|I|<\left|I_{1}\right| \leq\left|I_{2}\right| \leq \cdots \leq\left|I_{n}\right|$

$$
\begin{aligned}
1-\prod_{I \subset K, K \not \supset I \cup J} u_{K} & =1-u_{I} u_{I_{1}} \cdots u_{I_{n}}=1-u_{I}+u_{I}\left(1-u_{I_{1}} \cdots u_{I_{n}}\right) \\
& =\left(1-u_{I}\right)+u_{I}\left(1-u_{I_{1}}\right)+u_{I} u_{I_{1}}\left(1-u_{I_{2}}\right)+\cdots+u_{I} \prod_{i=1}^{n-1} u_{I_{i}}\left(1-u_{I_{n}}\right)
\end{aligned}
$$

Since $|I|<\left|I_{i}\right|$ for any $i$, we already know the $u$ equations for $I_{i}$ and we can find the equation for $u_{I}$.

Let us consider the $2 d$ example for which the relations are:

$$
\begin{aligned}
1-u_{1} u_{12} & =u_{0} u_{02}, & 1-u_{2} u_{12} & =u_{0} u_{01}, \\
1-u_{01} & =u_{2} u_{01} u_{12}, & 1-u_{02} & =u_{1} u_{02} u_{12}
\end{aligned}
$$

and the $u$ equations

$$
\begin{aligned}
1-u_{1} & =u_{0} u_{02}\left(1-u_{1} u_{01}\right), & 1-u_{2} & =u_{0} u_{01}\left(1-u_{2} u_{02}\right), \\
1-u_{01} & =u_{2} u_{01} u_{12}, & 1-u_{12} & =u_{0} u_{01} u_{02}
\end{aligned}
$$

which is different non-perfect set of $u$ equations for $\mathscr{P}_{2}$ unlike eq. (3.11)!

A nice fact about the $u$ equations we obtain is that $1-u_{I}$ are all at most linear and multi-term in $u_{I}$ for any $n$. However, they do not make the factorization manifest at the level of $u$ equations as we take some $u_{I} \rightarrow 0$ all incompatible $u_{J} \rightarrow 1$ but the $u$ equations corresponding to other $u$ 's are trivially satisfied thereby not reflecting the facets of $\mathscr{P}_{n}$ (as can be seen by taking $u_{12} \rightarrow 0$ in eq. (3.22)).

The $u$ equations for $\mathscr{P}_{n}$ similar to eqs. (3.11), (3.12) which do make factorization manifest are complicated polynomials in the corresponding $u$ 's. The most general $u$ equations can be conjectured to be:

$$
1-u_{I}=\left(1+h_{I, n}(u)\right) \prod_{J} u_{J}^{J \| I}
$$

where $J$ runs over all sets incompatible with $I, J \| I$ is a positive integer, called the "compatibility degree", and $h_{I, n}(u)$ is polynomial in $u_{I}$ such that $\left.h_{I, n}\right|_{u_{I}=0}=0$ which makes $\mathscr{P}_{n}$ binary as we proved using a different method in the previous section. But we do not have a closed form expression for $h_{I, n}(u)$ for all $n$ and $I$. We list some of them along with the $\mathscr{P}_{4}$ example in appendix (B).

Although the polynomial $h_{I, n}$ is very complicated for general $I$ and $n$, we can still say something about its "compatibility degree" $J|| I$ in eq. (3.23). Using eq. (3.10),

$$
1-u_{I}=1-\prod_{J \supset I} x_{J}^{(-1)^{|J|-|I|}}=\frac{\prod_{|J|-|I| \text { odd } ; \supset \supset I} x_{J}-\prod_{|J|-|I| \text { even; } J \supset I} x_{J}}{\prod_{|J|-|I| \text { odd } ; J \supset I} x_{J}}=: \frac{N_{I, n}(x)}{\prod_{|J|-|I| \text { odd } ; J \supset I} x_{J}},
$$


where $N_{I, n}(x)$ is a polynomial of degree $2^{|\bar{I}|-1}$ in $x$. It's easy to see that $x_{i}$ for $i \notin I$ is a factor of $N_{I, n}(x)$ by setting $x_{i}=0$, so we can define a new polynomial $f_{I, n}$ of degree $2^{|\bar{I}|-1}-|\bar{I}|$ with non-negative coefficients such that

$$
N_{I, n}(x)=f_{I, n}(x) \prod_{i \notin I} x_{i}
$$

Using eq. (3.19),

$$
\prod_{i \notin I} x_{i} / \prod_{|J|-|I| \text { odd; } J \supset I} x_{J}=\prod_{J \not \supset I} u_{J}^{|J|-|I \cap J|} \prod_{J \supsetneq I} u_{J}^{|J|-|I|-2^{|J|-|I|-1}} .
$$

Since $|J|-|I|-2^{|J|-|I|-1} \leq 0$ for $J \supsetneq I$, it's natural to rewrite eq. (3.24) as

$$
1-u_{I}=g_{I, n} \prod_{J \not \supset I} u_{J}^{|J|-|I \cap J|},
$$

where

$$
g_{I, n}=f_{I, n} / \prod_{J \supsetneq I} u_{J}^{2^{|J|-|I|-1}-(|J|-|I|)}=f_{I, n} / \prod_{\substack{J \supset I,|J|-|I| \text { odd } \\|J|-|I| \geq 3}} x_{J}
$$

It's easy to see that $g_{I, n}=1+h_{I, n}$ by taking $x_{I} \rightarrow 0$, where $h_{I, n}$ is a rational function of $x$ such that $\left.h_{I, n}\right|_{x_{I}=0}=0$. In fact,

$$
\left.N_{I, n}\right|_{x_{I}=0}=\prod_{|J|-|I| \text { odd } ; J \supset I} x_{J-I}=\prod_{i \notin I} x_{i} \prod_{\substack{J \supset I,|J|-|I| \text { odd } \\|J|-|I| \geq 3}} x_{J-I}=\left.\left.f_{I, n}\right|_{x_{I}=0} \prod_{i \notin I} x_{i} \Rightarrow g_{I, n}\right|_{x_{I}=0}=1 .
$$

Therefore,

$$
1-u_{I}=\left(1+h_{I, n}\right) \prod_{J \not \supset I} u_{J}^{|J|-|I \cap J|},
$$

and we conjecture that $h_{I, n}$ is a polynomial of $u$ with non-negative coefficients, and the "compatibility degree" is

$$
J|| I=|J|-|I \cap J| \text { for } I \not \subset J, \quad J|| I=0 \text { for } I \subset J .
$$

It's clear that any compatible $u_{J}$ for $J \subset I$ doesn't appear in the product because $I \cap J=J$. Note that it's not symmetric when $|I| \neq|J|$.

\subsection{Generalized permutohedra as binary geometries}

Inspired by the $u$ variables for permutohedra, we directly come up with the $u$ variables for generalized permutohedra. To this end, we first find all minimum extensions $I_{a}$ of $I$ in $\mathbf{B}$, that is there is no $J \in \mathbf{B}$ such that $I \subsetneq J \subsetneq I_{a}$, and we denote the collection of these extensions by $\mathscr{G}_{I}$. Then, the $u$ variables for the generalized permutohedron with the building set $\mathbf{B}$ are

$$
\log u_{I}:=\log x_{I}+(-1)^{k} \sum_{\varnothing \neq\left\{I_{a_{1}}, \ldots, I_{a_{k}}\right\} \subset \mathscr{G}_{I}} \log x\left(I_{a_{1}}, \ldots, I_{a_{k}}\right)
$$


where the sum is over all nonempty subsets of $\mathscr{G}_{I}$, and we introduce

$$
x\left(I_{a_{1}}, \ldots, I_{a_{k}}\right):=x_{I_{a_{1}} \cup \cdots \cup I_{a_{k}}}
$$

to avoid too many subscripts. Obviously, an equivalent form of eq. (3.26) is

$$
\log u_{I}:=(-1)^{k} \sum_{\left\{I_{a_{1}}, \ldots, I_{a_{k}}\right\} \subset \mathscr{G}_{I}} \log \left(x_{I}+x\left(I_{a_{1}}-I, \ldots, I_{a_{k}}-I\right)\right)
$$

Let us consider several simple examples to illustrate this construction:

(i) For a $n$-dimensional hypercube, $\mathscr{G}_{\{0\}}=\{[0,1]\}, \mathscr{G}_{\{i \neq 0\}}=\{[0, i]\}$ and $\mathscr{G}_{[0, i]}=\{[0, i+$ $1]\}$. This gives $u_{0}=x_{0} / x_{01}, u_{i}=x_{i} / x_{[0, i]}$ and $u_{[0, i]}=x_{[0, i]} / x_{[0, i+1]}$. These are indeed the $u$ variables for the $n$-dimensional hypercube since $u_{i+1}+u_{[0, i]}=1$ for $i \in[0, n-1]$.

(ii) For a $n$-dimensional associahedron,

$$
\mathscr{G}_{[i, j]}= \begin{cases}\{[i-1, j],[i, j+1]\} & \text { if } i \neq 0 \text { and } j \neq n \\ \{[0, j+1]\} & \text { if } i=0 \text { and } j \neq n \\ \{[i-1, j]\} & \text { if } i \neq 0 \text { and } j=n\end{cases}
$$

This reproduces the $u$ variables (2.2) for the $n$-dimensional associahedron.

(iii) For a $n$-dimensional cyclohedron, $\mathscr{G}_{[i, i+k]}=\{[i-1, i+k],[i, i+k+1]\}$. This reproduces the $u$ variables (2.4) for the $n$-dimensional cyclohedron.

(iv) For a $n$-dimensional permutohedron, $\mathscr{G}_{I}=\{I \cup\{j\} \mid j \in[0, n]-I\}$. This reproduces the $u$ variables (3.10) for the $n$-dimensional permutohedron.

For generalized permutohedra, as we have reviewed in section (3.1), $u_{I}$ and $u_{J}$ are incompatible if $I$ and $J$ are one of the following two cases:

(1) $I \cap J=\varnothing$ and $I \cup J \in \mathbf{B}$,

(2) $I \cap J \neq \varnothing, I, J$

while $u_{I}$ and $u_{J}$ are compatible if $I$ and $J$ satify one of the following three conditions:

(3) $J \subset I$,

(4) $I \subset J$,

(5) $I \cap J=\varnothing$ and $I \cup J \notin \mathbf{B}$.

We will follow the same argument as in the case of permutohedra: replacing $x_{i}$ with $\epsilon x_{i}$ for all $i \in I$ and taking $\epsilon \rightarrow 0$, then considering the behaviour of the other $u_{J}$ 's under this limit case by case. 
For case (1), some elements in $\mathscr{G}_{J}$ are subsets of $I \cup J$ since $I \cup J \in \mathbf{B}$. We denote the collection of such elements by $\mathscr{G}_{J}^{I \cup J}$. Then the logarithm of $u_{J}$ can be written as

$$
\log u_{J}=\sum_{\substack{\left\{J_{a_{1}}, \ldots, J_{a_{k}}\right\} \subset \mathscr{G}_{J}^{I \cup J} \\\left\{J_{b_{1}}, \ldots J_{b_{l}}\right\} \subset \mathscr{G}_{J}-\mathscr{G}_{J}^{I} J J}}(-1)^{k+l} \log \left(x_{J}+x\left(J_{a_{1}}-J, \ldots, J_{a_{k}}-J, J_{b_{1}}-J, \ldots, J_{b_{l}}-J\right)\right) .
$$

Since the second term in eq. (3.28) goes to $x\left(J_{b_{1}}-J-I, \ldots, J_{b_{l}}-J-I\right)$ under the limits of $x_{i} \rightarrow 0$ with $i \in I, \log u_{J}$ goes to 0 under this limit as in eq. (3.13). The whole argument can be carried over to case (2) by replacing $x_{J}$ with $x_{I \cap J}+x_{J-I}$.

For case (3), we again denote $\mathscr{G}_{J}^{I}$ as the collection of the elements in $\mathscr{G}_{J}$ which are subsets of $I$. After replacing $x_{i}$ with $\epsilon x_{i}$ for $i \in I$, we find the limit of $\log u_{J}$ as $\epsilon \rightarrow 0$ is

$$
\begin{gathered}
\lim _{\epsilon \rightarrow 0} \sum_{\substack{\left\{J_{a_{1}}, \ldots, J_{a_{k}}\right\} \subset \mathscr{G}_{J}^{I} \\
\left\{J_{b_{1}}, \ldots J_{b_{l}}\right\} \subset \mathscr{G}_{J}-\mathscr{G}_{J}^{I}}}(-1)^{k+l} \log \left(\epsilon x_{J}+\epsilon x\left(J_{a_{1}}-J, \ldots, J_{a_{k}}-J,\left(J_{b_{1}}-J\right) \cap I, \ldots,\left(J_{b_{l}}-J\right) \cap I\right)\right. \\
\left.\quad+x\left(J_{b_{1}}-I, \ldots, J_{b_{l}}-I\right)\right) \\
=\sum_{\substack{\left\{J_{a_{1}}, \ldots, J_{a_{k}}\right\} \subset \mathscr{G}_{J}^{I}}}(-1)^{k+l} \log \left(x_{J}+x\left(J_{a_{1}}-J, \ldots, J_{a_{k}}-J\right)\right) .
\end{gathered}
$$

Remarkably, these are $u$ variables for the generalized permutohedron with the building set $\mathbf{B}_{I}$ defined by (3.5).

Case (4) and (5) are quite trivial. For both cases, the limit behaviour of $u_{J}$ as $x_{I} \rightarrow 0$ can be simply obtained by replacing each $x_{K}$ in eq. (3.26) with $x_{K-I}$. Remarkably, these are the $u$ variables for the generalized permutohedron with the building set $\mathbf{B}_{\bar{I}}$ defined by (3.6).

Here we just showed that binary geometries exist for generalized permutohedra and gave the corresponding $u$ variables. The $u$ equations are quite complicated in general, and we only have closed formulas for $\mathscr{A}_{n}, \mathscr{B}_{n}$ and their several degenerations. For permutohedra, we have just conjectured the $u$ equations are of form (3.23). More generally, the $u$ equations don't even take the form of (3.23). For example, consider the generalized permutohedron with the building set

$$
\mathbf{B}=\{\{0\},\{1\},\{2\},\{3\},\{0,1,2\},\{1,3\},\{0,1,2,3\}\},
$$

the corresponding $u$ variables are

$$
\begin{aligned}
& u_{0}=\frac{x_{0}}{x_{012}}, \quad u_{1}=\frac{x_{1} x_{0123}}{x_{012} x_{13}}, \quad u_{2}=\frac{x_{2}}{x_{012}}, \quad u_{3}=\frac{x_{3}}{x_{13}} \\
& u_{012}=\frac{x_{012}}{x_{0123}}, \quad u_{13}=\frac{x_{13}}{x_{0123}} .
\end{aligned}
$$

The $u$ equations are ${ }^{6}$

$$
\begin{aligned}
u_{0}+u_{2}+u_{1} u_{13} & =1, & u_{1}+\left(u_{0}+u_{2}\right) u_{3} & =1, \\
u_{012}+u_{3} u_{13} & =1, & u_{13}+\left(u_{0}+u_{2}\right) u_{012} & =1 .
\end{aligned}
$$

We leave the study of compatible degrees and $u$ equations for general permutohedra to future work.

\footnotetext{
${ }^{6}$ If we introduce $\tilde{u}_{02}=u_{0}+u_{2}$, then $\tilde{u}_{02}$ together with the remaining $4 u$ 's form $\mathscr{A}_{2}$.
} 


\section{Discussions}

In this paper we introduce a large class of integrals, $\mathcal{I}_{\mathbf{B}}$, which can be regarded as a family of rigid stringy canonical forms for generalized permutohedra $\mathscr{P}(\mathbf{B})$, and we show that corresponding configuration spaces are always binary geometries. These rigid integrals satisfy the remarkable property that, at any "massless" pole, i.e. poles of the leading order $\Omega(\mathscr{P}(\mathbf{B}))$, the residue is given by a product of two such integrals, $\mathcal{I}_{\mathbf{B}_{L}} \times \mathcal{I}_{\mathbf{B}_{R}}$. Our results greatly extend the scope of binary geometries and associated integrals with such "perfect" factorization properties, which were originally thought to tie to the more special class of generalized associahedra for finite-type cluster algebras. For infinite families of cases as degenerations of associahedra and cyclohedra (which belong to both generalized permutohedra and generalized associahedra), we find additionally that the $u$ equations are "perfect" just like the case for cluster configuration spaces. Our preliminary investigations have opened up various new avenues to be explored, and we end with listing some open questions.

Generalized permutohedra vs. generalized associahedra. Our results provide an infinite number of examples generalizing ordinary string integrals (and cluster string integrals of type $B_{n}$ ), where the configuration space is determined by linear factors, or equivalently hyperplane arrangement. In this sense, it is fair to say that we have only explored a very special corner in the world of binary configuration spaces and associated stringy integrals. Even for cluster cases, it is unknown if the cluster configuration space of type $C_{n}$ can be realized by hyperplane arrangement (see also [25]), and for type $D_{n}$ and exceptional cases, the conjecture is that the spaces cannot be obtained this way [15]; we expect that these cases do not belong to integrals with "linear" factors. It would be highly desirable to look for a wider class of binary geometries which naturally incorporate both generalized permutohedra and generalized associahedra.

On the other hand, it would be interesting to study the configuration spaces for generalized permutohedra in a way similar to the cluster case. The $u$ equations for e.g. $\mathscr{P}_{d}$ are certainly worth further investigations, and one could ask if there might be any ana$\log$ /generalization of cluster algebra behind it. Another question concerns going beyond the positive part of the space since it is binary even in the complex case, and it would be nice to study complex space and connected components of the real space. For the cluster case, connected components are in bijection with sign patterns of the $u$ variables allowed by the so-called extended $u$ equations [15]. It would be highly desirable to see if one could obtain all the extended $u$ equations for e.g. $\mathscr{P}_{d}$, which can help us understand these questions better. Relatedly, the number of connected components and other interesting properties of the space can be inferred by counting the number of points in the space over a finite field; since we are dealing with hyperplane arrangement, the point count $N(p)$ is always a polynomial. Just to illustrate the counting with some examples, since $\mathscr{P}_{2}=\mathscr{B}_{2}$, the point count is $N(p)=(p-3)^{2}$, and if we plug in $p=-1$ we have $|N(-1)|=16$ connected components; we find for $\mathscr{P}_{3}, N(p)=(p-4)\left(p^{2}-10 p+26\right)$, thus there are 185 connected components, and for $\mathscr{P}_{4}, N(p)=p^{4}-30 p^{3}+345 p^{2}-1785 p+3485$ thus 5646 connected components. 
Moreover, since we have found a variety of such geometries with perfect $u$ equations from degenerating $\mathscr{A}_{n}$ and $\mathscr{B}_{n}$, it'd be very interesting to explore more cases with perfect $u$ equations from degenerations of other types, e.g. $\mathscr{C}_{n}$ and $\mathscr{D}_{n}$. Along this direction, an intriguing possibility is to classify binary geometries with perfect $u$ equations from degenerating cluster cases; since any product of such geometries still has perfect $u$ equations, this amounts to find all elementary, non-factorizing geometries with perfect $u$ equations. More importantly, what are the possible interpretations of these degenerations from cluster algebra; even for $\mathscr{A}_{n-3}$, what is the meaning of these degenerations of $\mathcal{M}_{0, n}$ and the string integrals?

Generalized string integrals and applications. One of the most pressing questions is to study further these stringy integrals and their potential applications to physics. We would like to understand why these integrals factorize at finite $\alpha^{\prime}$ just as cluster stringy integrals, though most of them are not related to cluster algebra. Recall that cluster stringy integrals of classical types are natural $\alpha^{\prime}$-deformation of bi-adjoint $\phi^{3}$ amplitudes through one-loop [17, 18], and in general the physical meaning of these integrals and their leading orders are unclear. A special class of generalized permutohedra, called Cayley polytopes, have natural interpretations as the sum of certain tree-level $\phi^{3}$ diagrams [26, 27]; for these cases, the stringy integrals provide a rigid $\alpha^{\prime}$-deformation of these $\phi^{3}$ "amplitudes". ${ }^{7}$ It would be interesting to see what role do generalize permutohedra play in the general story of formulating scattering amplitudes as differential forms [11, 13, 18, 30].

We have not touched various properties of these stringy integrals, such as recurrence relations, complex (and real) integrals with two different "orderings" and scattering (saddlepoint) equations and pushforward. It would be interesting to understand these points better, especially for the $\mathscr{P}_{d}$ case, which is completely symmetric in the labels. The scattering equations read

$$
\frac{S_{i}}{x_{i}}=\sum_{I \ni i} \frac{C_{I}}{x_{I}},
$$

which provide a one-to-one map from $\mathbf{x} \in \mathbb{R}_{\geq 0}^{d}$ to $\mathscr{P}_{d}$, and $\Omega\left(\mathscr{P}_{d}\right)$ is given by the pushforward via summing over the solutions. By plugging in $p=1$ in the point count $N(p)$, one can obtain the number of solutions (saddle points), which also gives the dimension of the space of integral functions [1]. For example, for $d=2,3,4$, we have 4,51,2016 solutions respectively.

One class of examples which are particularly interesting are the Stokes polytopes and Accordiohedra [31, 32] associated with planar $\phi^{p}$ amplitudes. A nice fact about Stokes polytopes $[32,33]$ is that any product of associahedra $\mathscr{A}_{i_{1}} \times \cdots \times \mathscr{A}_{i_{k}}$ is a Stokes polytope of dimension $n=i_{1}+\cdots+i_{k}$; more interestingly, degenerations of $\mathscr{A}_{3}$ with perfect $u$ equations correspond to $3 d$ Stokes polytopes. Furthermore every Stokes polytope is a Minkowski sum of associahedra [34] and thus it would be natural to consider them because these might provide a nice class of physically relevant examples which also play a role in classifying binary geometries with perfect $u$ equations.

\footnotetext{
${ }^{7}$ For other applications of generalized permutohedra to physics, see [28, 29].
} 
Beyond linear factors. Throughout the paper we have considered stringy integrals with linear factors whose Newton polytopes are coordinate simplices. In fact, one can discover more polytopes which also have perfect $u$ equations, once we consider non-linear factors. One particularly simple class of examples we discover concern polytopes that are Minkowski sum of $n$ line intervals and $n-1$ triangles, with Newton polynomials:

$$
\prod_{i=1}^{n}\left(1+x_{i}\right)^{-\alpha^{\prime} c_{i}} \prod_{i=1}^{n-1}\left(1+x_{i}+x_{i} x_{i+1}\right)^{-\alpha^{\prime} c_{i, i+1}}
$$

It turns out this gives a family of simple $n$-dimensional polytopes with $3 n-1$ facets and the number of vertices is given by the Pell number $P_{n}$ (recursively defined as $P_{n}=2 P_{n-1}+P_{n-2}$ with $P_{1}=1, P_{2}=2$ ). We refer to the polytope as $\mathscr{X}_{n}$.

The big polyhedron is a simplex, and we find that the $u$ variables take a very simple form:

$$
\begin{aligned}
& u_{1}=\frac{p_{n}}{q_{n}}, \quad u_{2}=\frac{p_{n-1} q_{n}}{p_{n-1, n}}, \quad u_{3}=\frac{p_{n-2} q_{n-1}}{p_{n-2, n-1}}, \ldots, \quad u_{n-2}=\frac{p_{1} q_{2}}{p_{12}}, u_{n-1}=\frac{p_{2} q_{3}}{p_{23}}, u_{n}=\frac{p_{3} q_{4}}{p_{34}}, \\
& u_{n+1}=\frac{1}{q_{1}}, \quad u_{n+2}=\frac{p_{12}}{q_{1} q_{2}}, \quad u_{n+3}=\frac{q_{1}}{p_{23}}, \ldots, \quad u_{3 n-1}=\frac{q_{n-1}}{p_{n-1 n}},
\end{aligned}
$$

where we denote $p_{i}:=x_{i}, q_{i}:=1+x_{i}$ and $p_{i+1}:=1+x_{i}+x_{i} x_{i+1}$. Remarkably, for any $n$, we have perfect $u$ equations which fall into three types viz. $1-u$ being the product of two, three or four $u$ 's. The first type has 4 equations:

$1-u_{1}=u_{3 n-2} u_{3 n-1}, 1-u_{n-2}=u_{n+1} u_{n+3}, 1-u_{n+1}=u_{n-2} u_{n+2}, 1-u_{3 n-1}=\left\{\begin{array}{l}u_{2} u_{3}, n=3 \\ u_{1} u_{4}, n=4 \\ u_{1} u_{2}, n \geq 5\end{array}\right.$,

with the corresponding facets being $\mathscr{X}_{n-1}$. The second type has $n-3$ equations

$1-u_{n+4}=u_{n} u_{n+2} u_{n+4} u_{n+6}, 1-u_{n+6+2 i}=u_{n-3-i} u_{n+4+2 i} u_{n+5+2 i} u_{n+8+2 i}$ for $i=0, \ldots, n-5$;

with the corresponding facets being $\mathscr{A}_{1}^{m} \times \mathscr{X}_{n-m-1}$. The third type has $2 n-2$ equations, but we do not yet have a complete classification of these facets. It would be nice to see if $\mathscr{X}_{n}$ 's are equivalent to some degenerations of $\mathscr{P}_{n}$, or if they are genuinely new binary geometries with perfect $u$ equations.

Stringy integrals at finite $\boldsymbol{\alpha}^{\prime}$. An important open question for stringy canonical form in general is to understand structures both at higher orders in the $\alpha^{\prime}$-expansion and even at finite $\alpha^{\prime}$. The leading order of (1.2) computes the canonical form of the corresponding polytope, which does not depend on the coefficients of the polynomials, but higher orders do, and it would be interesting to study $\alpha^{\prime}$ expansion for stringy integrals for e.g. permutohedra.

Moreover, having closed form expressions at finite $\alpha^{\prime}$ is certainly desirable. Our preliminary study already provides some examples, and let us look at a toy example which was considered in [1]:

$$
I\left(\alpha^{\prime}\right)=\int_{0}^{\infty} \int_{0}^{\infty} \frac{\mathrm{d} x}{x} \frac{\mathrm{d} y}{y}\left(\frac{1}{x}+\frac{1}{y}+x y\right)^{-\alpha^{\prime}} .
$$


We can use the symmetry in $x, y$ to make a symmetric change of variables to $u=x+y$ and $v=x y$, and the integral can be easily evaluated as

$$
I\left(\alpha^{\prime}\right)=\int_{0}^{\infty} \int_{0}^{\frac{u^{2}}{4}} \frac{2 \mathrm{~d} u \mathrm{~d} v}{v \sqrt{u^{2}-4 v}}\left(\frac{u}{v}+v\right)^{-\alpha^{\prime}}=\frac{\Gamma\left[\frac{\alpha^{\prime}}{3}\right]^{3}}{3 \Gamma\left[\alpha^{\prime}\right]} .
$$

This is in fact a special class of a large class of integrals which all correspond to the simplex case. By using the integral identity

$$
\int_{\mathbb{R}_{+}^{n}} \prod_{i=1}^{n} \frac{\mathrm{d} x_{i}}{x_{i}} x_{i}^{a_{i}} f\left(\sum_{i=1}^{n} x_{i}\right)=\frac{\prod_{i=1}^{n} \Gamma\left(a_{i}\right)}{\Gamma\left(\sum_{i=1}^{n} a_{i}\right)} \int_{0}^{\infty} f(\beta) \beta^{\sum_{i=1}^{n} a_{n}-1} \mathrm{~d} \beta,
$$

we can easily evaluate the following general integral for simplex:

$$
\begin{aligned}
& I\left(\alpha^{\prime}, X_{i}, c\right)=\int_{0}^{\infty} \cdots \int_{0}^{\infty} \prod_{i=1}^{n} \mathrm{~d} x_{i} x_{i}^{\alpha^{\prime} X_{i}-1}\left(b+\sum_{i=1}^{n} a_{i} x_{i}^{m_{i}}\right)^{-\alpha^{\prime} c} \\
& =\frac{b^{\alpha^{\prime}\left(\sum_{i=1}^{n} \frac{X_{i}}{m_{i}}-c\right)}}{\left(\prod_{i=1}^{n} m_{i}\right)\left(\prod_{i=1}^{n} a_{i}^{\alpha^{\prime} X_{i} / m_{i}}\right)} \int_{0}^{\infty} \cdots \int_{0}^{\infty} \prod_{i=1}^{n} \mathrm{~d} y_{i} y_{i}^{\frac{\alpha^{\prime} X_{i}}{m_{i}}-1}\left(1+\sum_{i=1}^{n} y_{i}\right)^{-\alpha^{\prime} c} \\
& =\frac{b^{\alpha^{\prime}}\left(\sum_{i=1}^{n} \frac{X_{i}}{m_{i}}-c\right)}{\left(\prod_{i=1}^{n} m_{i}\right)\left(\prod_{i=1}^{n} a_{i}^{\alpha^{\prime} X_{i} / m_{i}}\right)} B\left(\frac{\alpha^{\prime} X_{1}}{m_{1}}, \ldots, \frac{\alpha^{\prime} X_{n}}{m_{n}}, \alpha^{\prime}\left(c-\sum_{i=1}^{n} \frac{X_{i}}{m_{i}}\right)\right) \text {. }
\end{aligned}
$$

All stringy integrals (including the ones with non-linear factors) can in principle be evaluated as linear combination of A-hypergeometric functions with special arguments by identification the with a GKZ system [35, 36] (see also [37]). We shall leave the evaluation of more stringy integrals and related topics to future work.

\section{Acknowledgments}

We are grateful to Nima Arkani-Hamed, Thomas Lam, Hugh Thomas for stimulating discussions and collaborations on related projects. P.R. would like to thank the generous hospitality of the Institute of Theoretical Physics, Chinese Academy of Sciences, Beijing during a visit when part of this work was done. The research of S.H., Z.L. and C.Z. is supported in part by NSF of China under Grant No. 11947302 and 11935013.

\section{A Degenerations of $\mathscr{P}_{n}$ which lead to products}

In this section we consider degenerations of $\mathscr{P}$ give the most general products.

The maximal set of a building set $\mathbf{B}_{\max }$ is the set of all inclusion maximal elements of the building set $\mathbf{B}$. A building set $\mathbf{B}$ is connected if $\mathbf{B}_{\max }$ has a single element [24].

Theorem. If $\mathbf{B}_{1}, \mathbf{B}_{2}, \ldots \mathbf{B}_{n}$ are connected subsets of a building set $\mathbf{B}$ then the corresponding nestohedron $\mathscr{N}_{\mathbf{B}}$ is isomorphic to the product of the nestohedra $\mathscr{N}_{\mathbf{B}_{1}} \times \mathscr{N}_{\mathbf{B}_{2}} \cdots \times$ $\mathscr{N}_{\mathbf{B}_{n}}[24]$. 
The result follows as for any pair of connected building sets $\mathbf{B}_{i}, \mathbf{B}_{j}$ either $\mathbf{B}_{i} \cup \mathbf{B}_{j}=\varnothing$ in which case $\mathcal{I}_{\mathbf{B}_{i} \cup \mathbf{B}_{j}}=\mathcal{I}_{\mathbf{B}_{i}} \times \mathcal{I}_{\mathbf{B}_{j}}$ or $\mathbf{B}_{i} \cup \mathbf{B}_{j}=\{k\}$ in this we can rescale the $x_{l} \rightarrow x_{k} x_{l}$ for $l \in \mathbf{B}_{j}$ to decouple the integrals. Thus we always get $\mathcal{I}_{\mathbf{B}}=\mathcal{I}_{\mathbf{B}_{1}} \times \mathcal{I}_{\mathbf{B}_{2}} \cdots \times \mathcal{I}_{\mathbf{B}_{n}}$.

Using the above result we construct a degeneration of $\mathscr{P}_{n}$ which are the most general products i.e., $\prod_{p_{i}} X_{p_{i}}$ such that $\sum_{i=1}^{k} p_{i}=n$ by dividing the $(n+1)$ singlets into $k$ parts

$$
\left[0, p_{1}\right], \quad\left[p_{1}, p_{1}+p_{2}\right], \quad \ldots, \quad\left[\sum_{i=1}^{k-1} p_{i}, \sum_{i=1}^{k} p_{i}=n\right]
$$

and using them to construct the building sets $\mathbf{B}_{p_{i}}$ of $X_{p_{i}}\left[\sum_{j=1}^{(i-1)} p_{j}, \sum_{j=1}^{i} p_{j}\right]$.

Example. Degenerations of $\mathscr{P}_{4}$ into $\mathscr{A}_{1}^{4}, \mathscr{A}_{1}^{2} \times \mathscr{A}_{2}, \mathscr{A}_{1} \times \mathscr{A}_{3}, \mathscr{A}_{2}^{2}, \mathscr{A}_{1}^{2} \times \mathscr{B}_{2}, \mathscr{A}_{1} \times \mathscr{B}_{3}, \mathscr{A}_{1} \times$ $\mathscr{P}_{3}, \mathscr{A}_{2} \times \mathscr{B}_{2}$ and $\mathscr{B}_{2}^{2}$.

For $\mathscr{A}_{1}^{4}$ the building set is $\{0,1,2,3,4,01,12,23,34\}$ where $\{0,01\},\{1,12\},\{2,23\},\{3,34\}$ generate the $4 \mathscr{A}_{1}$ 's which we denote as $\mathscr{A}_{1}(0,1) \times$ $\mathscr{A}_{1}(1,2) \times \mathscr{A}_{1}(2,3) \times \mathscr{A}_{1}(3,4)$.

For $\mathscr{A}_{1}^{2} \times \mathscr{A}_{2}$ the building set is $\{0,1,2,3,4,01,12,23,34,234\}$ generated by $\mathscr{A}_{1}(0,1) \times$ $\mathscr{A}_{1}(1,2) \times \mathscr{A}_{2}(2,3,4)$ 's.

For $\mathscr{A}_{1} \times \mathscr{A}_{3}$ the building set is $\{0,1,2,3,4,01,12,23,34,123,234,1234\}$ generated by $\mathscr{A}_{1}(0,1) \times \mathscr{A}_{3}(1,2,3,4)$.

For $\mathscr{A}_{2}^{2}$ the building set is $\{0,1,2,3,4,01,12,23,34,012,234\}$ generated by $\mathscr{A}_{2}(0,1,2) \times$ $\mathscr{A}_{2}(2,3,4)$

For $\mathscr{A}_{1}^{2} \times \mathscr{B}_{2}$ the building set is $\{0,1,2,3,4,01,12,23,34,24,234\}$ generated by $\mathscr{A}_{1}(0,1) \times \mathscr{A}_{1}(1,2) \times \mathscr{B}_{2}(2,3,4)$ 's.

For $\mathscr{A}_{1} \times \mathscr{B}_{3}$ the building set is $\{0,1,2,3,4,01,12,23,34,14,123,234,134,124,1234\}$ generated by $\mathscr{A}_{1}(0,1) \times \mathscr{B}_{3}(1,2,3,4)$.

For $\mathscr{A}_{1} \times \mathscr{P}_{3}$ the building set is $\{0,1,2,3,4,01,12,23,34,14,13,24,123,234,134$, $124,1234\}$ generated by $\mathscr{A}_{1}(0,1) \times \mathscr{P}_{3}(1,2,3,4)$.

For $\mathscr{A}_{2} \times \mathscr{B}_{2}$ the building set is $\{0,1,2,3,4,01,12,23,34,24,012,234\}$ generated by $\mathscr{A}_{2}(0,1,2) \times \mathscr{B}_{2}(2,3,4)$

For $\mathscr{B}_{2}^{2}$ the building set is $\{0,1,2,3,4,01,12,02,23,34,24,012,234\}$ generated by $\mathscr{B}_{2}(0,1,2) \times \mathscr{B}_{2}(2,3,4)$.

We can similarly find a degeneration of $\mathscr{P}_{n}$ which would correspond to any product.

\section{B Some $u$ equations of $\mathscr{P}_{n}$}

Some of the $u$ equations for the $n$-dimensional permutohedron are: for $|I|=n$ or $n-1$ we have

$$
1-u_{I}=\prod_{J \not \supset I} u_{J}^{|J|-|I \cap J|}
$$

and for $|I|=n-2$,

$$
1-u_{I}=\left(1+\prod_{J \supset I} u_{J}\right) \prod_{J \not \supset I} u_{J}^{|J|-|I \cap J|}
$$


The other $u$ equations are not two or three-term equations, and in general it has the form

$$
1-u_{I}=\left(1+h_{I, n}(u)\right) \prod_{J \not \supset I} u_{J}^{|J|-|I \cap J|}
$$

where $h_{I, n}(u)$ is conjectured to be a polynomial of $\left\{u_{J} \mid J \supset I\right\}$ such that $\left.h_{I, n}(u)\right|_{u_{I}=0}=0$.

Let us write down the $\mathscr{P}_{4}$ example:

$$
\begin{aligned}
u_{0} & =\frac{x_{0} x_{012} x_{013} x_{014} x_{023} x_{024} x_{034} x_{01234}}{x_{01} x_{02} x_{03} x_{04} x_{0123} x_{0124} x_{0134} x_{0234}}, & u_{01} & =\frac{x_{01} x_{0123} x_{0124} x_{0134}}{x_{012} x_{013} x_{014} x_{01234}}, \\
u_{012} & =\frac{x_{012} x_{01234}}{x_{0123} x_{0124}}, & u_{0123} & =\frac{x_{0123}}{x_{01234}}
\end{aligned}
$$

The $u$ equations are

$$
\begin{aligned}
& \qquad \begin{aligned}
1-u_{0}= & u_{1} u_{2} u_{3} u_{4} u_{12}^{2} u_{13}^{2} u_{14}^{2} u_{23}^{2} u_{24}^{2} u_{34}^{2} u_{123}^{3} u_{124}^{3} u_{134}^{3} u_{234}^{3} u_{1234}^{4}\left(1+h_{\{0\}, 4}\right), \\
1-u_{01}= & u_{2} u_{3} u_{4} u_{02} u_{03} u_{04} u_{12} u_{13} u_{14} u_{23}^{2} u_{24}^{2} u_{34}^{2} u_{023}^{2} u_{024}^{2} u_{034}^{2} u_{123}^{2} u_{124}^{2} u_{134}^{2} u_{234}^{3} u_{0234}^{3} u_{1234}^{3} \\
& \times\left(1+h_{\{0,1\}, 4}\right), \\
1-u_{012}= & u_{3} u_{4} u_{03} u_{04} u_{13} u_{14} u_{23} u_{24} u_{34}^{2} u_{013} u_{014} u_{023} u_{024} u_{034}^{2} u_{123} u_{124} u_{134}^{2} u_{234}^{2} u_{0134}^{2} u_{0234}^{2} u_{1234}^{2}, \\
1-u_{0123}= & u_{4} u_{04} u_{14} u_{24} u_{34} u_{014} u_{024} u_{034} u_{124} u_{134} u_{234} u_{0124} u_{0134} u_{0234} u_{1234}
\end{aligned} \\
& \text { where }
\end{aligned}
$$

$$
\begin{aligned}
h_{\{0,1\}, 4}= & u_{01} u_{012} u_{013} u_{014} u_{0123} u_{0124} u_{0134}, \\
h_{\{0\}, 4}(u)= & u_{0} u_{01}^{3} u_{02} u_{03} u_{04} u_{012}^{3} u_{013}^{3} u_{023} u_{024} u_{034} u_{0123}^{2} u_{0124}^{2} u_{0134}^{2} u_{0234} u_{014}^{3} \\
& +u_{0} u_{01} u_{02} u_{03} u_{04} u_{012}^{2} u_{013}^{2} u_{023} u_{024} u_{034} u_{0123}^{2} u_{0124}^{2} u_{0134}^{2} u_{014}^{2} \\
& +u_{0}^{2} u_{01}^{2} u_{02}^{2} u_{03}^{2} u_{04}^{2} u_{012}^{2} u_{013}^{2} u_{023}^{2} u_{024}^{2} u_{034}^{2} u_{0123} u_{0124} u_{0134} u_{0234}^{2} u_{014}^{2} \\
& +u_{0} u_{01}^{2} u_{02} u_{03} u_{04} u_{012}^{2} u_{013}^{2} u_{023} u_{024} u_{034} u_{0123} u_{0124} u_{0134} u_{0234}^{2} u_{014}^{2} \\
& +u_{0} u_{01} u_{02} u_{03} u_{04} u_{012} u_{013} u_{023} u_{024} u_{034} u_{0234} u_{014} .
\end{aligned}
$$

The other $u$ variables and $u$ equations can be obtained by permutation of the indices. The 10 facets of $\mathscr{P}_{4}$ obtained by setting $u_{i}, u_{i j k l} \rightarrow 0$ are all $\mathscr{P}_{3}$ and the 20 facets obtained by setting $u_{i j}, u_{i j k} \rightarrow 0$ are all $\mathscr{A}_{1} \times \mathscr{P}_{2}$.

Open Access. This article is distributed under the terms of the Creative Commons Attribution License (CC-BY 4.0), which permits any use, distribution and reproduction in any medium, provided the original author(s) and source are credited.

\section{References}

[1] N. Arkani-Hamed, S. He and T. Lam, Stringy canonical forms, arXiv:1912.08707 [INSPIRE].

[2] L. Nilsson and M. Passare, Mellin transforms of multivariate rational functions, J. Geom. Anal. 23 (2011) 24.

[3] C. Berkesch, J. Forsgård and M. Passare, Euler-Mellin integrals and A-hypergeometric functions, Michigan Math. J. 63 (2014) 101. 
[4] E. Panzer, Hepp's bound for Feynman graphs and matroids, arXiv:1908.09820 [INSPIRE].

[5] F.C.S. Brown, Multiple zeta values and periods of moduli spaces $M_{0, n}(R)$, Annales Sci. Ecole Norm. Sup. 42 (2009) 371 [math/0606419] [INSPIRE].

[6] K. Aomoto and M. Kita, Theory of hypergeometric functions, Springer, Japan (2011).

[7] S. Mizera, Scattering amplitudes from intersection theory, Phys. Rev. Lett. 120 (2018) 141602 [arXiv: 1711.00469] [INSPIRE].

[8] P. Mastrolia and S. Mizera, Feynman integrals and intersection theory, JHEP 02 (2019) 139 [arXiv: 1810.03818] [INSPIRE].

[9] S. Mizera and A. Pokraka, From infinity to four dimensions: higher residue pairings and Feynman integrals, JHEP 02 (2020) 159 [arXiv: 1910.11852] [INSPIRE].

[10] F. Brown and C. Dupont, Single-valued integration and double copy, arXiv:1810.07682 [INSPIRE].

[11] N. Arkani-Hamed, Y. Bai, S. He and G. Yan, Scattering forms and the positive geometry of kinematics, color and the worldsheet, JHEP 05 (2018) 096 [arXiv: 1711.09102] [INSPIRE].

[12] F. Cachazo, S. He and E.Y. Yuan, Scattering of massless particles: scalars, gluons and gravitons, JHEP 07 (2014) 033 [arXiv:1309.0885] [INSPIRE].

[13] N. Arkani-Hamed, Y. Bai and T. Lam, Positive geometries and canonical forms, JHEP 11 (2017) 039 [arXiv : 1703.04541] [INSPIRE].

[14] F. Cachazo, S. He and E.Y. Yuan, Scattering of massless particles in arbitrary dimensions, Phys. Rev. Lett. 113 (2014) 171601 [arXiv:1307.2199] [INSPIRE].

[15] N. Arkani-Hamed, S. He, T. Lam and H. Thomas, Binary geometries, generalized particles and strings, and cluster algebras, arXiv:1912.11764 [INSPIRE].

[16] N. Arkani-Hamed, S. He and T. Lam, Cluster configuration spaces of finite type, arXiv: 2005.11419 [INSPIRE].

[17] G. Salvatori, 1-loop amplitudes from the halohedron, JHEP 12 (2019) 074 [arXiv: 1806.01842] [INSPIRE].

[18] N. Arkani-Hamed, S. He, G. Salvatori and H. Thomas, Causal diamonds, cluster polytopes and scattering amplitudes, arXiv:1912.12948 [INSPIRE].

[19] S. Fomin and A. Zelevinsky, Y systems and generalized associahedra, Ann. Math. 158 (2003) 977 [hep-th/0111053] [INSPIRE].

[20] N. Arkani-Hamed, T. Lam and M. Spradlin, Positive configuration space, arXiv:2003.03904 [INSPIRE].

[21] A. Postnikov, Permutohedra, associahedra and beyond, Int. Math. Res. Not. 2009 (2009) 1026 [math.C0/0507163].

[22] V. Bazier-Matte, G. Douville, K. Mousavand, H. Thomas and E. Yıldırım, ABHY associahedra and Newton polytopes of F-polynomials for finite type cluster algebras, arXiv: 1808.09986 [INSPIRE].

[23] J.-Y. Shi, The Kazhdan-Lusztig cells in certain affine Weyl groups, Lect. Notes Math. 1179 (1986) 312.

[24] A. Postnikov, V. Reiner and L. Williams, Faces of generalized permutohedra, Documenta Math. 13 (2008) 207 [math. C0/0609184]. 
[25] Z. Li and C. Zhang, Moduli space of paired punctures, cyclohedra and particle pairs on a circle, JHEP 05 (2019) 029 [arXiv: 1812.10727] [INSPIRE].

[26] X. Gao, S. He and Y. Zhang, Labelled tree graphs, Feynman diagrams and disk integrals, JHEP 11 (2017) 144 [arXiv: 1708.08701] [INSPIRE].

[27] S. He, G. Yan, C. Zhang and Y. Zhang, Scattering forms, worldsheet forms and amplitudes from subspaces, JHEP 08 (2018) 040 [arXiv: 1803.11302] [INSPIRE].

[28] N. Early, Generalized permutohedra, scattering amplitudes, and a cubic three-fold, arXiv: 1709.03686 [INSPIRE].

[29] N. Early, Generalized permutohedra in the kinematic space, arXiv:1804.05460 [INSPIRE].

[30] S. He and C. Zhang, Notes on scattering amplitudes as differential forms, JHEP 10 (2018) 054 [arXiv: 1807.11051] [INSPIRE].

[31] P. Banerjee, A. Laddha and P. Raman, Stokes polytopes: the positive geometry for $\phi^{4}$ interactions, JHEP 08 (2019) 067 [arXiv:1811.05904] [INSPIRE].

[32] P. Raman, The positive geometry for $\phi^{p}$ interactions, JHEP 10 (2019) 271 [arXiv: 1906.02985] [INSPIRE].

[33] F. Chapoton, Stokes posets and serpent nests, Discrete Math. Theor. Comput. Sci. 18 (2015) [arXiv: 1505.05990]

[34] Y. Baryshnikov, On stokes sets, in New developments in singularity theory, Springer, Dordrecht, The Netherlands (2001), pg. 65.

[35] M. Saito, B. Sturmfels and N. Takayama, Gröbner deformations of hypergeometric differential equations, Springer, Berlin, Heidelberg, Germany (2000).

[36] I. Gelfand, M. Kapranov and A. Zelevinsky, Generalized Euler integrals and A-hypergeometric functions, Adv. Math. 84 (1990) 255.

[37] L. de la Cruz, Feynman integrals as A-hypergeometric functions, JHEP 12 (2019) 123 [arXiv: 1907.00507] [INSPIRE]. 